\title{
Co-movement and Lead-Lag Relationship Between Green Bonds and Renewable Energy Stock Markets: Fresh Evidence from the Wavelet-Based Approach
}

\section{Nana Liu}

China University of Mining and Technology - Xuzhou Campus: China University of Mining and Technology

Chuanzhe Liu ( $\nabla$ rdean@cumt.edu.cn )

China University of Mining and Technology https://orcid.org/0000-0002-5911-1296

\section{Research}

Keywords: Green bonds, renewable energy markets, wavelet coherence, Causality, COVID-19

Posted Date: November 18th, 2020

DOI: https://doi.org/10.21203/rs.3.rs-105937/v1

License: (c) This work is licensed under a Creative Commons Attribution 4.0 International License.

Read Full License 
Co-movement and lead-lag relationship between green bonds and renewable energy

1 School of Economics and Management, China University of Mining and Technology, Xuzhou 221116, China; liunana1004@cumt.edu.cn (N.L.);

* Correspondence: rdean@cumt.edu.cn; Tel.: +86-516-83590168

\section{Abstract:}

8 Background: A recent study in Nature Climate Change shows that due to reduced human activities during the Coronavirus disease 2019 (COVID-19) pandemics, daily global emissions of carbon dioxide decreased by $17 \%$ from the average level in 2019 . With the gradual recovery of economic activity and human energy consumption, the emissions of greenhouse gas and pollution would rise again. Green bonds are considered a crucial tool to release climate finance. The green bond market can act as an essential bridge between capital providers (i.e. institutional investors) and sustainable assets (i.e. renewable energy). between green bonds and global and sector renewable energy stock markets in the time and frequency horizons. We apply continuous wavelet, wavelet coherence, and line and nonline causality approaches on data during the period 2010-2020, coincidentally including the COVID-19 pandemic.

Results: (1) Green bonds and renewable energy markets show evidence of a similar pattern based on the wavelet power spectrum, which shows high price volatility at small and medium scales, especially during periods of turbulence and crisis. (2) The dynamic connection between green bonds and renewable energy returns is weak (strong) on the short (long) time-scale. However, on medium-term time scales, the dependence between them is significant only during turbulent periods, such as the European Sovereign Debt Crisis 2012 (ESDC) and the COVID-19 pandemic. (3) With regard to causality, our results show unidirectional and bidirectional linear (non-linear) causality at low and high frequencies. Moreover, our finding reveals the fact regarding the lead - lag relationship that, most of the time and frequencies, no one market necessarily dominates the other.

Conclusion: Our findings provide several remarkable policies and practical implications for market regulators and investors. Institutional investors can benefit by including green bonds in their portfolios to decrease their climate change risk and improve their environmental, social, and corporate governance rating in the portfolio. Considering that 
the dependence between green bonds and renewable energy stock prices varies over various time scales, investors with different investment horizons should make diverse investment portfolio and hedging choices. The finding is also relevant for formulating green finance policies and supporting renewable energy investments. Policy decisions on the transition of energy to a decarbonized economy should consider the consequences for green bonds, which are also critical for the transition to a climate-resilient economy.

\section{Keywords:}

Green bonds; renewable energy markets; wavelet coherence; Causality; COVID-19

\section{Background}

A recent study in Nature Climate Change [1] shows that due to reduced human activities during the Coronavirus disease 2019 (COVID-19) pandemics, daily global emissions of carbon dioxide decreased by $17 \%$ from the average level in 2019. Carbon emissions at the end of May 2020 were close to the level of 2006. With the gradual recovery of economic activity and human energy consumption, the emissions of greenhouse gas and pollution would rise again. To implement fully the Paris Agreement 2015, \$1.5 trillion in green financing would be required every year until 2030. Attracting low-carbon investment in green energy program has been a significant challenge and needs a major shift [2-4]. This shift requires government policy to reallocate financial resources, and one of the most effective ways to attract investment in more sustainable development projects is to promote green bonds [5].

Green bonds are considered a crucial tool to release climate finance [6, 7]. Green bonds are attracting increasing interest in Asia and around the world as an alternative source of financing for low-carbon projects $[5,8]$. Green bonds are used differently compared with general bonds [9]. The green bond process is only used to finance the low-carbon projects, while general bonds are allowed to finance any legal project. Investors are well aware of the present challenges of climate change, and green bonds could fund our path to a more sustainable world [7]. The green bond market can act as an essential bridge between capital providers and sustainable assets. The green bond market has developed rapidly, growing from $\$ 3.4$ billion in 2012 to $\$ 156$ billion in 2017 . To raise additional finance for clean production projects, the European Investment Bank and the World Bank were the first to issue green bonds in 2007 and 2008, respectively. The market is now gradually diversifying 
in the types of issuer, geographic region and usage of funds. For example, 55 issuers from different countries/regions had issued green bonds by the end of 2019. International green bonds involve a total of 496 issuers, including multilateral development banks, sovereign countries, local governments, government-supported institutions, financial institutions, and non-financial enterprises [10].

Although the progress of green bond markets has been impressive, such markets still have opportunities for further growth and improvement [11]. The confirmed financing of green bonds occupies only $17 \%$ of the reported unlabeled climate-related bonds. To achieve further market growth, the coordinated action among many stakeholders is needed. Policymakers can help with the supply of green bonds (i.e. adopting cutting-edge climaterelated green bond standards) and provide supportive policies to promote the growth of the renewable energy sector. Public capital providers could contribute to the elimination of renewable assets and support green bonds by providing seed capital, demonstration issuance and capacity building. Institutional investors may help by aligning their internal capabilities and investment objectives with long-term sustainability requirements. Other stakeholders, such as rating agencies, financial institutions, and retail investors, could also play an essential role in strengthening the green bond market and advancing global energy transition. Analyzing the risk spillover between green bonds and renewable energy markets on determining the market development of green bonds and their role in hedging portfolio risk is critical. Therefore, understanding how green bonds move with the stock prices of renewable energy is an essential concern for investors and policymakers.

To the best of our knowledge, this work is the first that focuses on the co-movement and lead-lag relationship between green bonds and renewable energy and considers the time and frequency scales simultaneously. Although wavelet analysis methods have been used in several studies on the connection between energy and financial asset returns[12-17], this study is the first attempt to analyze the interaction between green bonds and renewable energy using the continuous (discrete) wavelet transformation method. The primary advantage of the wavelet approach is that it allows us to distinguish short- and long-term investor behaviors. More precisely, market investors trade on a variety of time scales (expressed as frequencies) that range from seconds to years mainly because they have various degrees of beliefs, goals, preferences and institutional constraints, as well as distinct levels of information acceptance and risk tolerance. For example, agents with 
shorter investment maturities, such as day traders or hedge funds, are more interested in the short-term actions of the market. Alternatively, other agents, such as large institutional investors, are more concerned with long-term market behavior. Therefore, an appropriate frequency band would help to understand better the co-movement of green bonds and renewable energy stocks at different frequency levels.

What should the relationship between the renewable energy stock prices and green bond yields be? There is a view that there should be a negative correlation using a present -value model. For instance, an increase in the discount rate in the future is expected to lead to a fall in share prices and an increase in long-term interest rates. However, there may also be a positive correlation, as changes in long-term interest rates may be related to information about the future dividend stream of the stock [18]. Several contradictory assumptions may predict a co-movement between these two green assets. This hypothesis is closely related to the theoretical arguments about the relationship between stock and conventional bonds [19], although the issuance of green bonds is ostensibly driven by the "green bond principle". There are the following representative hypotheses about risk spillovers between stock and bonds markets: (1) Financial risk contagion: In the absence of a material change in economic fundamentals, adverse shocks in one market are automatically transmitted to the other, leading to movements in the same direction, especially in times of extreme risk [20]; (2) Risk hedging needs: when the price of an asset deviates too much from its real value, hedgers will shift more of their positions to other safe assets to reach the target hedge ratio level [21]; (3) Asset substitution: Assuming that stocks and bonds are two perfectly competing assets, if the disclosure of relevant information helps increase the price of stocks, investors will be incentivized to convert bonds into stocks in their portfolio; if the information is more favorable to bonds, investors will replace their stock holdings with bonds [22]. When hypothesis (1) is confirmed, the stock and bond markets exhibit a "linkage effect"; when hypothesis (2) and hypothesis (3) are confirmed, there is usually a "seesaw effect" between the two. The co-movement between the two markets can also be explained by the above three hypotheses since the renewable energy stock and green bond markets are subordinate to the stock and bond markets, respectively. We expect financial contagion between the green bonds and renewable energy markets because, as an important source of funding for renewable energy companies, when the overall green bond market improves, investors expect the renewable energy markets to strengthen as well. 
To this end, we analyze (i) dynamic co-movement and the lead-lag relationship between cross time-scale by applying cross-wavelet coherence and phase analysis, and (ii) the causality between green bonds and renewable energy returns by using (discrete) wavelet methods combined with linear and non-linear causality tests. We find that the interaction between green bonds and renewable energy returns is weak in the short time scale and that this weakness persists throughout the sampling period. In the long run (512 days-), green bonds are closely linked to the renewable energy market, despite differences between the global and sectoral indices. However, on medium-term time scales, the degree of dependence between these two markets is high only during turbulent periods. Concerning causality, our results show unidirectional and bidirectional linear (non-linear) causality at low and high frequencies. Moreover, our results reveal the fact regarding the lead-lag relationship that, at most time and frequency, no one market necessarily dominates the other.

This study investigates the idiosyncratic characteristics of return connections between green bonds and renewable energy markets. We examine these linkages because they are important for investment and risk management decisions. For example, portfolio managers often transfer funds from stocks to bonds when they expect stock market returns to decline. Reducing risk through this transfer depends on the linkages between the stock and bond markets. If cross-market asset returns are highly correlated, bonds would not provide the risk aversion that investors need. And if cross-market asset returns are negatively correlated, the possibility exists for long-term asset portfolios. Exploring the dynamics of the correlation between stock and bonds markets can provide theoretical support for the practice of asset allocation by institutional investors such as investment funds and insurance funds. Linkages between markets should also be taken into account when formulating regulatory policy, for example, market regulators would consider these linkages when assessing the effects of proposed policy changes.

This work provides a novel insight into green investment from a new perspective and contains at least four contributions on green bonds and renewable energy research. First, we use a continuous wavelet transformation method to distinguish between short- and longterm investor behavior in green bonds and renewable energy stocks. This aspect is important for investors who act at different time scales and over different periods. Indeed, from the perspective of portfolio diversification, green portfolio managers are more interested in higher frequency asset price linkages. In other words, they are concerned 
about short-term movement. However, others are more interested in lower frequencies (i.e., longer-term time scale). Second, using the frequency domain to understand the two main green assets better and choose the incentive policy that suits them is useful for policymakers. Third, a non-linear Granger causality model is applied to analyze further the relationship between green bonds and renewable energy over different time horizons. Fourth, we also use the most recent dataset, which happens to include the COVID-19 epidemic period, resulting in extreme market volatility. As a result, we add an interesting period for the green bond and renewable energy markets.

The rest of the paper is organized as follows. Section 2 reviews the literature on green bonds and renewable energy. Section 3 introduces the data and reports a preliminary analysis. Section 4 outlines the methodology. Section 5 presents the empirical results. Section 6 offers primary conclusions and implications.

\section{Literature review}

Many researchers have focused on the relationship between green bonds and other markers. Pham [23] first provided evidence that the labelled green bond market is more volatile than the "unlabeled" bonds by using the Standard \& Poor Co. (S\&P) Green Bond Index. In a comparable study, Bachelet et al. [24]confirmed that green bonds issued by institutional issuers have higher liquidity than gray bonds. Reboredo [25] investigated the co-movement between green bonds and financial markets. This finding suggested a strong linkage between the treasury and corporate bond markets, and a weak connection between stock and energy commodity markets. Likewise, Reboredo and Ugolini [26] employed the value-at-risk (VaR) approach and discovered the price correlation between green bonds and financial markets. This study provided evidence that the green bond market is closely related to the fixed income and currency markets, resulting in a considerable price spillover effect and a negligible reverse effect. However, the green bonds market is weakly linked to these markets, such as stock, energy, and high-yield corporate bond markets. The research of Reboredo et al. [27] further provided a similar result to that of Reboredo and Ugolini [26] by using wavelet coherence methods; their finding suggested a strong connectedness between green bonds and treasury and corporate bonds over different time horizons and in the European Union (EU) and the United States (US); they found a weak linkage between green bonds and high-yield corporate bonds and stock and energy assets in the short and 
193 long term. Recently, Jin et al. [28] made the first attempt to investigate the relationship

194 between carbon futures and green bonds, as well as other three markets; this finding 195 supported the evidence of the fact that the green bond market is the effective hedge for 196 carbon futures and has performed well in periods of crisis. The relationship between green 197 bonds and other markets could be affected by factors such as financial market volatility, 198 economic policy uncertainty, oil prices, and positive and negative news reports on green 199 bonds. Moreover, the attitudes and measures at all levels of governments can directly 200 influence the green bond markets [29]. All of this prior literature invariably argued that the 201 relationship between green bonds and energy was weak, with only considering the whole 202 energy market, and failed to consider the renewable energy market.

203 It is noteworthy that the empirical relationship between green bonds and renewable 204 energy has been considerably neglected. To the best of our knowledge, only a few articles 205 provide an overview of the relationship between them $[5,8,30]$. For example, Liu et al. 206 [32] investigated the dynamic dependence between green bonds and clean energy markets 207 through a time-varying copula model. Hammoudeh et al. [33] analyzed the time-varying 208 relationship between green bonds and other assets such as clean energy, CO2 emission 209 allowance prices and other markets using a novel time-varying Granger causality test with 210 July 30, 2014 to February 10, 2020 as the sample period. Nguyen et al. [34]consider the 211 clean energy market in the study of the relationship between green bonds and other asset 212 markets, and through the rolling window wavelet analysis method, the association between 213 green bonds and clean energy is high, while the correlation with other markets such as 214 stocks and commodities market is weak. Le, et al. [35] explore the return and volatility 215 spillover effects between green bonds and financial technology and cryptocurrencies from 216 both time domain and frequency domain perspectives, and the results show that green 217 bonds can be used as good hedging assets.

218 Given that green bonds offer significant funding for renewable energy projects [31], the 219 intrinsic connection between green bonds and the renewable energy market deserves 220 further exploration. This study is the first attempt to examined the co-movement and lead221 lag relationship between green bonds and renewable energy markets across different time 222 horizons by applying (discrete) wavelet methods, and linear and non-linear causality tests. 223 It would fill in the gaps for the empirical research of green bonds and renewable energy.

\section{3. Data}


We conduct an empirical analysis of co-movement and lead-lag relationship between green bonds and renewable energy stock prices on a range of time scale. In this case, we consider the daily data of three global and three sectoral renewable energy indices, as well as green bond indices. Referring to the research of Reboredo [36], we chose the S\&P Dow Jones Green Bonds Index (hereafter GB) to indicate the global green bond market. Green bonds refer specifically to the bonds utilized to finance environmental projects. Given the diversity of the renewable energy market, it is crucial to consider different energy companies. Ugolini et al. [37] and Rezec and Scholtens [38] found a suitable basis for estimating the renewable energy market by targeting six indices for different sectors of renewable energy .

The three global indices include the following: (a) The Wilder Hill Clean Energy Index (ECO) is comprised of 42 companies focused on renewable energy technologies. The selection of stocks and sectors included in this index is based on their relevance to clean energy, technological advances, and the elimination of pollution $[39,40]$. (b) The S\&P 500 Global Clean Index (GCE) is a weighted index comprised of more than 30 companies from around the world in the clean energy production or equipment industries, including clean energy production companies and equipment and technology companies [41]. (c) The European Renewable Energy Index (ERIX), which includes 30 of the largest European clean energy companies specifically involved with biomass, solar, geothermal, marine, water and wind energy [42]. The sectoral indices are as follows: (d) The ISE Global Wind Energy Index (WIND) is designed to track the performance of companies by offering listed products and services in the wind energy industry. (e) The MAC Global Solar Equity Index (SOLAR) is a diversified solar energy index that includes all solar energy technology, operations and financing across the value chain, and related solar equipment. (f) The S\&P Renewable Energy and Clean Technology Index (TECH) measures the key performance of companies that focus on green technology and sustainable infrastructure solution.

The data for all indices are obtained from the Bloomberg database. Our sample period runs from March 29, 2010 to June 30, 2020 and totals 2670 daily observations, which coincidentally cover periods of major market turmoil, such as European Sovereign Debt Crisis 2012 (ESDC) and the COVID-19 period. We obtain the daily return series for all variables by the logarithmic difference method. Figure 1 depicts the trend of fluctuations in a multi-pair time series, the trajectory of which may suggest a positive and strong 
correlation between green bonds and renewable energies. Fig.2 reports the dynamics of green bond and renewable energy index returns. Interestingly, all indicators have a similar path, especially the energetic vibration during the ESDC crisis and the COVID-19 pandemic, although the amplitude of the wave is different.

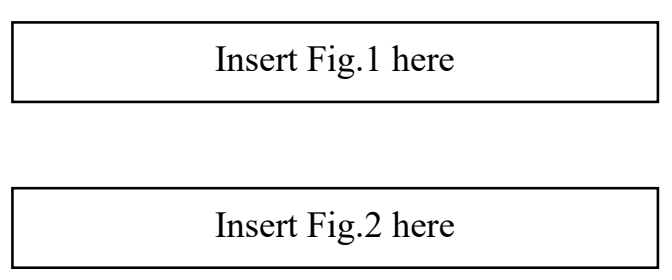

Table 1 reports the descriptive statistical characteristics of the returns on green bonds and six renewable energy assets. The mean values of the seven asset returns are all close to zero. The standard deviations indicate that the volatility of all return series except GB and SOLAR are similar. SOLAR returns have maximum and minimum extremes of 0.120 and -0.15 , respectively. Therefore, the risk of volatility is the highest. Conversely, a positive mean of GB has the smallest standard deviation (0.004) and is thus a safe investment. Moreover, this study finds that all variables are negatively skewed, with ECO and GCE having the highest negative skewness values $(-0.75)$ with the most obvious risk of collapse. At the same time, the excess peaks (higher than standard 3) indicate that all variables are characterized by a spiky thick-tailed distribution, which is also confirmed by the Jarque-Bera test. Ljung-Box and Autoregressive Conditional HeteroskedasticityLagrange Multiplier tests explicitly detect a correlation in the return series. The results of the unit root tests (i.e., ADF, PP and KPSS) reject the null hypothesis of the existence of a unit root at the $1 \%$ significance level, suggesting that all return series are stationary. Finally, Pearson correlation coefficients demonstrate that all renewable energy indices are positively correlated with green bond yields.

\section{Insert Table 1 here}

\section{Methodology}

\subsection{Continuous wavelet transform (CWT)}

The wavelet is a function constructed from a single wavelet known as the mother wavelet, 
which is a real-valued squared productive function given by the following:

$$
\psi_{\tau, s}(t)=\frac{1}{\sqrt{s}} \psi\left(\frac{t-\tau}{s}\right),
$$
where $\frac{1}{\sqrt{s}}$ is the normalization constant that ensures the unit variance of the wavelet, and $\tau$ and $s$ are the position and scale parameters that determine the precise position of the wavelet and wavelet expansion or stretching, respectively.

Each wavelet can help characterize different data. In this paper, we utilize Morlet wavelets, which are often used in the economic field, to obtain amplitude and phase. The Morlet wavelet consists of a Gaussian window Fourier transform in which the sine and cosine vibrate at the core frequency and is calculated as follows:

$$
\psi(\mathrm{t})=\pi^{-\frac{1}{4}} e^{i w_{0} t} e^{\frac{-t^{2}}{2}},
$$

299 where $\pi^{-\frac{1}{4}}$ is the standardized factor that ensures that the wavelet has a unit of energy, $300 e^{\frac{-t^{2}}{2}}$ as a Gaussian envelope with unit standard deviation, and $e^{i w_{0} t}$ denote a complicated sinusoidal curve. In the present study, we set $w_{0}=6$ to represent the appropriate compromise between time and frequency localization.

The CWT $W_{x}(s)$ is a useful tool that enables to analyze time evolution along with the frequency and is described as follows:

$$
W_{x}(s)=\int_{-\infty}^{\infty} x(t) \frac{1}{\sqrt{s}} \psi *\left(\frac{t}{s}\right),
$$

where $*$ denotes complex conjugation and the proportionality parameter $s$ determines whether the wavelet can detect a higher or lower component of the sequence $x(t)$, which is possible when the tolerance condition is satisfied.

\subsection{Cross-wavelet power, wavelet coherence and phase differences}

The application of continuous wavelet analysis in financial and economic fields is mainly focused on the multi-scale analysis of univariate variables. However, to examine the co-movement and lead-lag relationship between two variables in time-frequency domains simultaneously, cross-wavelet transform is also required.

The cross-wavelet transform explores the interdependence between green bonds $x(t)$ and renewable energy $y(t)$ in a different frequency space, which can be formulated as follows:

$$
W_{n}^{X Y}(\tau, s)=W_{n}^{X}(\tau, s) * W_{n}^{Y}(\tau, s)
$$


where $W_{n}^{X}(\tau, s)$ and $W_{n}^{Y}(\tau, s)$ denote CWT of $\mathrm{x}(\mathrm{s})$ and $\mathrm{y}(\mathrm{s})$, respectively and * represents the complex conjugate.

As opposed to the power wavelet, crossed wavelet power (XWP) represents the local covariance in time and frequency for each sequence, and the formula is as follows:

$$
X W P^{X}(\tau, s)=\left|W_{n}^{X Y}(\tau, s)\right| .
$$

Wavelet coherence $R_{n}^{2}(s)$ is also an important method for assessing the common movement between green bonds and renewable energy in the time-frequency space. It generates a quantity between 0 and 1 (a correlation coefficient), where 0 denotes a weak inter-correlation and 1 means a strong interaction. $R_{n}^{2}(s)$ is given by:

$$
R_{n}^{2}(s)=\frac{s^{-1}\left|W_{n}^{X Y}(s)\right|^{2}}{\left(s^{-1}\left|W_{n}^{X}(s)\right|^{2}\right)\left(s^{-1}\left|W_{n}^{Y}(s)\right|^{2}\right)},
$$

where $s$ is the smoothing element of time and scale. As suggested by Torrence and Compo [43], Monte Carlo simulation methods can be used to perform statistical inference.

Moreover, whether the nexus between green bonds and renewables is positive or negative and whether a lagging or lagging relationship exists can be measured by the phase difference. Torrence and Webster [44] gave the following definition:

$$
\varphi_{x y}(s)=\tan ^{-1}\left(\frac{\zeta\left(s^{-1} W_{n}^{X Y}(s)\right)}{\xi\left(s^{-1} W_{n}^{X Y}(s)\right)}\right),
$$

where $\zeta$ denotes the imaginary component and $\xi$ represents the real part. When $\varphi_{x y}(s)=0$, the two series are in the same period (in-phase), which implies that they are positively interconnected. When $\varphi_{x y}(s)=\pi$ or $-\pi$, the two series will be moved with a $180^{\circ}$ (out of phase), which suggests a negative association. Fig.3 provides a summary of the different types of phases, represented by the direction and angle of the arrows.

\subsection{Discrete wavelet transform (DWT)}

However, the CWT will create redundant information, which leads to inefficient analysis. Therefore, the DWT is performed to account for specific time-frequency conditions adequately.

Parameters s and $\tau$ are discretized as $\mathrm{s}=2^{-j}, \tau=2^{-j k}, j, k \in Z$, and the definition of the wavelet function becomes the following: 


$$
\psi_{j, k}(t)=2^{\frac{j}{2}} \psi\left(2^{j} t-k\right), j, k \in Z .
$$

Thus, the DWT is specified as follows:

$$
W_{x}(j, k)=\int x(t) \overline{\psi_{\jmath, k}(t)} d t, j=0,1,2, \ldots, k \in Z .
$$

A multi-resolution analysis is introduced to allow the decomposition of the return series into different scales. The decomposition of $\mathrm{x}(\mathrm{t})$ is calculated as follows:

$$
\mathrm{x}(\mathrm{t})=\sum_{k} s_{J, k} \phi_{J, k}(t)+\sum_{k} d_{J, k} \psi_{J, k}(t)+\sum_{k} d_{J-1, k} \psi_{J-1, k}(t)+\cdots+\sum_{k} d_{1, k} \psi_{1, k}(t),(10
$$

where $\phi$ and $\psi$ are two fundamental functions referred to as father and mother wavelets, respectively, which describe the low and high-frequency section of the sequence. The parameters $s_{J, k}, d_{J, k}, \ldots, d_{1, k}$ are wavelet transform factors that determine the response of the corresponding wavelet function to the overall spectrum. Thus, using the J-level multiresolution decomposition analysis, the time series $\mathrm{x}(\mathrm{t})$ is represented as:

$$
\mathrm{x}(\mathrm{t})=S_{J}(t)+D_{J}(t)+D_{J-1}(t)+\cdots+D_{1}(t)
$$

where $S_{J}(t)$ is the base function or residual, and frequency components $D_{J}(t)$ provides time-frequency details for the short, medium, or long term. In this study, we create eight separate multi-resolution levels based on sample data to filter the financial data appropriately and correctly (see Gençay et al., 2002,2005). The decomposition results in eight specific time-frequency include the following: (1) the highest frequency component, D1, indicates a time scale of 2 days (daily impact); (2) component D2 represents a time scale of 4 days (weekly impact); and (3) components D3, D4, D5, D6, D7, and D8 measure medium- and long-term variations from 8 days to 256 days.

\subsection{Linear and non-linear Granger causality tests}

After converting the green bond and renewable energy variables into proportional components, we used the linear and non-linear Granger causality tests. Granger causality intends to examine whether the current and lagged values of a variable can significantly contribute to the prediction of the future value of another variable. The linear causality for two stationary series $\mathrm{X}$ and $\mathrm{Y}$ is calculated by a bivariate vector autoregressive (VAR):

$$
\begin{aligned}
& X_{t}=\alpha_{0}+\sum_{i=1}^{n} \alpha_{i} X_{t-i}+\sum_{j=1}^{q} \beta_{j} Y_{t-j}+\varepsilon X, t, \\
& Y_{t}=\beta_{0}+\sum_{i=1}^{n} \alpha_{i} X_{t-i}+\sum_{j=1}^{q} \beta_{j} Y_{t-j}+\varepsilon Y, t,
\end{aligned}
$$


where $\mathrm{X}$ and $\mathrm{Y}$ are stable time series, and $\mathrm{n}$ and $\mathrm{q}$ are the lag lengths of $\mathrm{X}$ and $\mathrm{Y}$, respectively. The null hypothesis in the Granger causality test is that $\mathrm{y}$ does not cause $\mathrm{x}$, which is indicated by $H_{0}: \beta_{1}=\cdots=\beta_{q}=0$. The contrast hypothesis is $H_{1}: \beta_{i} \neq 0$ for at least one $\mathrm{j}$. The test statistic has a standard $\mathrm{F}$ distribution, in which the degrees of freedom are (n, T$\mathrm{n}-\mathrm{q}-1)$ and $\mathrm{T}$ is the number of observations.

One weakness of linear causality measures is their failure to accommodate nonlinearities in time series price dynamics [45-48]. Several authors have formulated a variety of nonparametric tests for Granger's non-causal hypothesis. Baek and Brock [49] developed a nonparametric statistical method based on correlation integration. Hiemstra and Jones [50] introduced an adapted non-linear causality model that relaxes the assumptions of Baek and Brock [49] about independent and identical distribution levels and mutual independence. Furthermore, Diks and Panchenko [51] explored a new non-linear Granger test, which is widely used to evaluate economic and energy market data. Thus, in the present study, the non-linear Granger approach proposed by Diks and Panchenko [51] is used to test the non-linear relationship between green bonds and the renewable energy market.

Assuming two strictly stationary time series $X_{t}$ and $Y_{t}$, if the past and current values of $X_{t}$ contain additional information about the future value of $Y$ that is not included in the past and current $Y_{t}$ values, then $X_{t}$ strictly Granger leads to $Y_{t} . F_{X, t}$ and $F_{Y, t}$ denote the set of past information for $X_{t}$ and $Y_{t}$ before time $\mathrm{t}+1$, respectively, and order $\sim$ indicates the equivalent distribution. The time series $X_{t}$ is the Granger causality of $Y_{t}$ when the following conditions are met:

$$
\left(Y_{t+1}, \ldots, Y_{t+k}\right)\left|\left(F_{X, t}, F_{Y, t}\right) \sim\left(Y_{t+1}, \ldots, Y_{t+k}\right)\right| F_{X, t},
$$

where $\mathrm{k} \geq 1$ is the forecast border. Given the lag vectors $X_{t}^{L_{X}}=\left(X_{t-L_{X}+1}, \ldots, X_{t}\right)$ and $Y_{t}^{L_{y}}=\left(Y_{t-L_{Y}+1}, \ldots, Y_{t}\right),\left(L_{X}, L_{Y} \geq 1\right)$, the null hypothesis supposes that the past observations of $X_{t}^{L_{X}}$ include no additional information about $\mathrm{Y}(\mathrm{t}+1)$ compared with those of $Y_{t}^{L_{y}}$.

5

$$
H_{0}: Y(t+1)\left|\left(X_{t}^{L_{x}} ; Y_{t}^{L_{y}}\right) \sim Y(t+1)\right| Y_{t}^{L_{y}}
$$

For the strictly stationary time series, Eq. 15 follows the invariant distribution of the $\left(L_{x}+\right.$ $\left.L_{y}+1\right)$ - dimensional vector $W_{t}=\left(X_{t}^{L_{x}}, Y_{t}^{L_{y}}, Z_{t}\right)$, where $Z_{t}=Y_{t+1}$. To keep the following presentation and discuss denote ion compact, we dropped the time subscript and 
assume $\mathrm{Lx}=\mathrm{Ly}=1$. The conditional distribution of $\mathrm{Z}$, given $(X, Y)=(x, y)$, was assumed to be the same as that of $\mathrm{Z}$ given $\mathrm{Y}=\mathrm{y}$. Thus, we redefined the Eq.14 as follows:

$$
\frac{f_{X, Y, Z}(x, y, z)}{f_{Y}(y)}=\frac{f_{X, Y}(x, y)}{f_{Y}(y)} \cdot \frac{f_{Y, Z}(y, z)}{f_{Y}(y)} .
$$

Following Eq. 16, for each fixed value of $\mathrm{y}, \mathrm{X}$ and $\mathrm{Z}$ are conditionally independent of $\mathrm{Y}$ $=\mathrm{y}$. Thus, the modified null hypothesis indicates that the following equation is satisfied:

$$
\mathrm{q} \equiv \mathrm{E}\left[f_{X, Y, Z}(x, y, z) f_{Y}(y)-f_{Y, Z}(y, z)\right]=0 .
$$

Let $\hat{f}_{W}\left(W_{i}\right)$ as the local density estimator of the random vector $\mathrm{W}$ at $W_{i}$,

$$
\hat{f}_{W}\left(W_{i}\right)=\frac{\left(2 \varepsilon_{n}\right)^{-d_{W}}}{(n-1)} \sum_{j, j \neq 1} I_{i j}^{W},
$$

where $I_{i j}^{W}=I\left(\left\|W_{i}-W_{j}\right\|<\varepsilon_{n}\right), \mathrm{I}(\cdot)$ represents the indicator function, and $\varepsilon_{n}$ stands for the bandwidth parameter related to the $\mathrm{N}$ number of samples. Given an estimate of the local density function, the following test statistics is constructed:

$$
T_{n}\left(\varepsilon_{n}\right)=\frac{n-1}{n(n-2)} \cdot \sum_{i}\left(\hat{f}_{X, Y, Z}\left(X_{i}, Y_{i}, Z_{i}\right) \hat{f}_{Y}\left(Y_{i}\right)-\hat{f}_{X, Y}\left(X_{i}, Y_{i}\right) \hat{f}_{Y, Z}\left(Y_{i}, Z_{i}\right)\right) .
$$

For $L_{x}=L_{y}=1$, when $\varepsilon_{n}=C n^{-\beta}\left(C>0, \frac{1}{4}<\beta<\frac{1}{3}\right)$, the statistic $T_{n}\left(\varepsilon_{n}\right)$ satisfies the following conditions:

$$
\sqrt{n} \frac{\left(T_{n}\left(\varepsilon_{n}\right)-q\right)}{S_{n}} \stackrel{D}{\rightarrow} N(0,1),
$$

where $\rightarrow \mathrm{D}$ is the convergence of the distribution and $S_{n}$ stands for the estimate of the asymptotic variance of $T_{n}(\cdot)$.

\section{Results and discussion}

\subsection{Evidence from continuous wavelet analysis}

The unconditional correlations (see the last row of Table 1) provide proof that green bonds are correlated with renewable energy. This study spans a long period. Thus, understanding how these correlations develop over time and explore whether dependency varies with frequencies (i.e., whether more distinctive interdependencies exist over longer or shorter investment horizons) would be interesting. The wavelet coherence method allows us to investigate the correlations in time and frequency fields simultaneously.

Figure 3 displays the CWT power spectrum of the green bond and renewable energy return series. The horizontal axis represents time, and the vertical axis stands for the frequencies, which are converted to units of time (day) for ease of interpretation. In terms 
of technology, as in previous related studies [13, 52, 53], we used Monte Carlo simulations to assess statistical significance. The bold black contour represents the regions that are significant at the $5 \%$ level. The black line of the curve indicates the cone of influence (i.e., the area affected by the edge effect). The warmer (cooler) color represents the more intense (smooth) fluctuations in the time-frequency domain.

First, the results of the wavelet power spectrum may help us identify common islands between the green bond yield and the renewable energy return index on different time horizons. Closely inspecting Fig.4, the green bonds and renewable energy show a significant power in the same period and frequency from 2010 to 2012 and early 2020 on the scale of 1 day to 256 days. Therefore, these markets show evidence of the same pattern based on the wavelet power spectrum. The wavelet power spectra show high price volatility at small and medium scales, especially during periods of turbulence and crisis, such as the 2012 ESDC, the Fed's interest rate hike policy in 2016, and the recent COVID-19 pandemic. This finding may suggest that the relationship between the green bond market and renewables is much solid during the period of crisis.

Moreover, the high-power region is also apparent in Fig. 4 at the lowest scales (1-day scale to 32-day scale) for all the renewable energy markets over the entire research period. This observation reveals that a significant period of price volatility in the renewable energy markets exists at low scales, which may be attributed to the anxiety of investors and market traders to intervene in these markets in the short term. The mix of cool and warm colors across the graph shows no clear evidence of structural change in the series.

Furthermore, the wavelet power spectrum plot shows, in the upper left (early 2010 to 2012) and top right corner (early 2020) of the wavelet power spectrum for green bond returns, obvious red and yellow islands, indicating high power. However, in other regions, in short-, medium-, and long-term scales, the plot shows intense blue islands (deficient power). This finding may demonstrate that, except for the crisis periods, the price of green bonds showed stable fluctuations.

\section{Insert Fig. 4 here}

The cross-wavelet power is applied to calculate the wavelet coherence coefficients for the local covariance between the green bond and renewable energy markets at various times 
and frequencies. The wavelet coherence diagram (Fig. 6) visually displays the strength of the local relation between the green bonds and the renewable energy market in the timefrequency space. It can also reveal the leading-lagging relationship (correlation) of the phase-difference information between the two. The arrow pointing in the right direction indicates that the two are in phase, that is, they are positively correlated and vice versa. The arrow pointing up means the green bond is ahead of the renewables and vice versa.

Fig. 5 displays the results of the cross-wavelet transformation between green bonds and renewables, showing the local association between these indicators at different times and frequencies. The cross-wavelet correlation characteristics between green bonds and the six renewable energy sources are similar. Moreover, the evidence in Fig. 5 also suggests that the covariance is gradually weakening as the frequency decreases, which means that the correlation between green bonds and renewable energy returns is more affected by shortterm shocks than by long-term and sustained changes. When cross wavelet coherence is particularly high, specific periods (2010-2012 and early 2020) and particular frequencies (high) can be identified. Furthermore, the impact strength of green bonds on renewable energies decreases over time such that, in the following years of the sample (2017-2019), covariance decreases across all time horizons and all variables. In addition, the available information on the phases (indicated by the arrows) suggests that the association between green bonds and renewable energies is not uniform across time scales because the arrows point upwards, to the right, and to the left on different time scales.

Insert Fig. 5 here

Moreover, the wavelet coherence and phase difference are applied to detect the lead-lag relationship of green bond-renewable energy pairs. In the wavelet coherence diagram (Fig. 6), the color grade orders from warmer (higher cohesion) to cooler (lower cohesion). The lowest coherence is close to 0 (dark blue), implying a perfect negative cohesion, whereas the highest cohesion is close to +1 (dark red), which means a perfect positive cohesion. The horizontal axis shows time, and the vertical represents the period, converting it to units of time (days).

The visual inspection of Fig. 6 reveals several interesting findings. We have identified that these markets share the same pattern over the long-term horizon. Green bond and 
504 renewables are weakly reliant at high frequencies, and this weakness persists throughout 505 the entire sampling period. However, green bonds have become less dependent (fewer red 506 islands) on the renewable energy market after 2012. In the short-term, the correlation 507 between green bonds and renewable energies is time-varying. As a result, the coherence is 508 unstable with time (a few continuous red or blue islands). However, at lower frequencies 509 (512 days-), a persistent red area is seen at the lowest bottom of the wavelet correlation 510 plot, except for $\mathrm{TECH}$, where the long-term scale relationship weakens, suggesting that 511 green bonds are strongly correlated with the renewable energy market (only except for 512 TECH). Fig. 6 also shows that the red islands increased massively on intermediate time 513 scales during the 2010-2012 crisis and the COVID-19 pandemic, suggesting that the 514 connection between the green bonds and the renewable energy indices is only highly 515 dependent on the medium scale during the turmoil. This strong link between green bonds 516 and renewables during the crisis can be explained by the following fact: the fundamentals 517 of common action behavior (medium and long-term investors) are compromised during the 518 whole non-calm period.

519 Furthermore, we also observe that the phase shown by the arrows in Fig. 6 is pointing to 520 the right most of the time and frequencies, suggesting that the local relevance is positive 521 and that renewables do not dominate the price of green bonds. The low frequency (512 522 days-) of all pairs shows that the global renewable energy indices (i.e., ECO and GCE) and 523 the sector renewables (i.e., WIND) have changed from pointing to the upper right to the 524 right since 2013, which provides some rough and brief evidence that, early in the sample, 525 green bonds are ahead of changes in renewable energy prices. However, as time evolves, a 526 positive correlation and no lead-lag relationship are observed between the two. For the 527 ERIX index, the phase points to the upper right on the low-frequency (512-day-) scale, 528 indicating that green bond prices are leading the renewable energy prices. This evidence is 529 consistent throughout the sample period. However, for the sectoral renewable energy index 530 (i.e., SOLAR and TECH), at the low frequency (512 days-), the arrows point mainly to the 531 lower right, which shows that green bond prices are lagging renewable energy prices. Our 532 empirical results provide new evidence for co-movement between green bond and 533 renewables. 
5.2. Evidence on causality

5.2.1 Linear Granger causality analysis between green bonds and renewable energy markets

In the linear Granger causality test, a bivariate VAR model of the market is developed, and the Akaike information criterion (AIC) criterion is used to determine the optimal lag order. Tables 2 and 3 show the results of the linear Granger causality test between the green respectively. For the original returns series, the empirical results demonstrate bidirectional linear Granger causality between the green bonds and renewable energy market at the 5\% significance level, except for CEO and SOLAR, which have unidirectional linear Granger causality from green bonds to the renewable energy markets. Although renewable energies are based on supply and demand of the market, green bond prices still have an important effect on renewable energy markets.

On multiple time scales, the green bonds and renewable energy markets display different linear Granger causality. For the short-term (D1), the linear Granger non-causality is rejected at the 5\% significance, indicating unidirectional linear Granger causality running from the renewable energy market to the green bond market. It also shows that the volatility of renewable energy prices in the short term has a linear effect on the green bond market. Nonetheless, for the ERIX and TECH markets, which are exceptions, we do not find linear causality between green bonds and TECH on short-run time scales.

However, on medium time scales D2-D7 (more than one week and less than one year, excluding non-working days), the linear Granger test results support that, in most cases, the two markets are linearly correlated at the 5\% significance level. Using the linear Granger causality test, bidirectional Granger causality is observed between green bonds and renewable energy markets for most modes on intermedium time scales (i.e., D2, D3, and D4), as well as unidirectional Granger causality running from the green bond market to renewable energy market for D6. Moreover, for D7, a linear Granger relationship is not found in these two markets, except for ERIX and WIND. These results have two possible reasons. First, the formation of D2-D6 is influenced by factors with medium-term implications, such as shocks from major episodes or structural alterations in renewable 
energy policy that could alter the whole renewable energy system and lead to comparable market changes, thereby increasing spillovers between green bonds and renewable energy markets. Second, due to the long duration of the volatility, eliminating the impact of these factors on market shocks in the short term is difficult, and the impact can spread from one market to the other. Overall, over the medium-term horizon, a significant bidirectional linear Granger is observed between the green bonds and the renewable energy market. As for the long-run trends, linear Granger causality tests suggest that the two return series are expected to increase in approximately 216 days (i.e., almost one year, excluding nonworking days) over a reasonably long time horizon in both directions. Therefore, despite their distinctive characteristics, the long-term trends in both market returns follow similar patterns, slowly fluctuating around the zero mean.

Insert Table 2 here
Insert Table 3 here
markets
The non-linear Grange models to detect the non-
linear relationship between the green bonds and renewable energy markets at original and decomposition data. Table 5 gives the values of the statistics $T$ and $P$. According to the research of Diks and Panchenko [51], the parameter $\mathrm{C}$ of the bandwidth is 8, and the theoretical optimal rate of $\beta$ is $2 / 7$, and with reference to Yu et al. [54], we set the optimal bandwidth $\varepsilon_{n}$ to 1.5 based on our sample size.

For the original data, we discovered that the Granger causality was rejected at a $5 \%$ significance level on the return sequence, that is, bidirectional non-linear Granger causality is observed between the green bonds and the renewable energy market. This observation is different from the unidirectional linear Granger relationship running from the renewable energies (i.e., ECO and SOLAR) to the green bond market. For short-term time scales, the non-linear Granger non-causality between green bonds and renewable energy markets is rejected at a 5\% significance level, indicating bidirectional non-linear Granger causality between the two markets. The short-term fluctuations of the green bond and the renewable 
energy markets have a non-linear interaction with each other. This finding is different from the linear Granger causality test, which only found that most of the short-term fluctuations in the renewable energy market would affect the short-term fluctuations in the green bond market.

For intermediate time scales (i.e., more than one week and less than one year, excluding non-working days), the Granger test results find evidence supporting a non-linear relationship between the two markets in most cases, with a significance level of $5 \%$ (see Panel B-G of Table 5). For the majority of cases, the results of the non-linear Granger causality test are consistent with that of the linear Granger causality test. Nonetheless, some discrepancies remain between the linear and non-linear test results. For instance, non-linear bidirectional Granger causality at D5 and D6 can be statistically demonstrated at the 5\% significance level, but we do not identify linear causality running from the green bonds to the renewable energy market. The possible reasons are the drivers of these patterns on intermediate time scales (i.e., major events and policy changes, which are well-documented) can lead to structural breakdowns in the renewable energy market. Given this structural fracture, the two return series exhibit visible non-linear characteristics on the medium time scales, and the traditional linear Granger causality model may be difficult to capture. On the contrary, the bidirectional Granger relationship on the medium-term can be effectively examined by the non-linear Granger causality test.

Focusing on the long term D8 (i.e., more than one year, excluding non-working days), the test findings support the evidence of non-linear Granger causality between the two return series (See Panel H in Table 5). This result is quite different from the linear Granger causality test, which identifies the linear bidirectional Granger causality over a long period. The primary explanation could be attributed to the simple, linear, and low-level complexity characteristic of the two long-term market trends. Given that the two series move slowly on a smooth curve without significant structural breaks, the connection mechanism between them may be following a simple linear relationship rather than a complex nonlinear relationship. In general, our findings reveal that the green bond market is closely associated with the renewable energy market. 


\section{Conclusion and implications}

Assessing the co-movement between green bonds and renewable energy markets has become one of the most pioneering and interesting topics to elaborate on the potential benefits of green bonds portfolio diversification and risk management. In this study, we provided fresh evidence for the time-frequency dynamic co-movement and lead-lag relationship between green bonds and six renewable energy markets from March 29, 2010 to June 30, 2020 by using (discrete) wavelet analysis, wavelet coherency, cross wavelet methods, and linear and non-linear causality tests. Several important pieces of evidence can be concluded as follows.

Green bonds and renewable energy markets show evidence of a similar pattern based on the wavelet power spectrum, which shows high price volatility at small and medium scales, especially during periods of turbulence and crisis. The wavelet coherence analysis, which shows that the common movement between the pair of return sequences depends on time and frequency, is greatly impacted by the financial crisis, which cannot be captured by traditional time series techniques. We provided evidence that the dynamic interaction between green bonds and renewable energy returns is weak in the short-term and that this weakness persists throughout the sampling period although green bonds have become less dependent (fewer red islands) on the renewable energy market after 2012. In the long time scale (512 days-), green bonds are strongly correlated with the renewable energy market despite slight differences between the global and sectoral indices. However, on mediumterm time scales, the connection between the green bond market and the renewable energy market is highly dependent only during turbulent periods, such as the 2010-2012 ESDC and the COVID-19 pandemic. With regard to causality, our results show unidirectional and bidirectional linear (non-linear) causality at low and high frequencies. Moreover, our finding reveals the fact regarding the lead - lag relationship that, most of the time and frequencies, no one market necessarily dominates the other.

Our findings provide several remarkable policies and practical implications for market regulators and investors. Specifically, the fact that the price of green bonds is less volatile than that of renewable energy stocks provides a new investment target for investors. Investors consider green bonds as investment assets and/or hedge portfolio risk while holding investment positions in energy stock assets. Institutional investors can also benefit 
more by including green bonds in their portfolios because doing so would decrease their climate change risk and improve their environmental, social, and corporate governance rating in the portfolio.

Considering that the dependence between green bonds and renewable energy stock prices varies over various time scales, investors with different investment horizons should make diverse investment portfolio and hedging choices. Our evidence shows that green bonds and renewable energy assets are weakly correlated in the short run. Therefore, shortterm investors could use green bonds as a hedge against renewable energy investments to reduce risk volatility. On the medium-term scale, given that the relationship would further strengthen during turbulent periods, investors should focus on the risk transfer between the two markets and design appropriate portfolio ratios to reduce and diversify portfolio risk.

We found evidence that green bonds and clean energy markets are positively correlated and co-moved on long time scales. Moreover, the results of the Granger test indicate bidirectional Granger causality between green bonds and renewable energy stocks, which prevents investors from taking advantage of hedging. However, investors could design their portfolios using the evidence of linear and non-linear causality because these two markets would use each other for useful information in determining their future values. Particularly, information from other markets should be carefully considered when forecasting market prices for green bonds or renewable energy.

Our finding is also relevant for formulating green finance policies and supporting renewable energy investments. In particular, when renewable energy and green bond prices move up (down) together, public clean energy funding can have an impact on renewable energy companies. This influence may result in a price externality for green bonds. Likewise, the removal of supportive policies (e.g., subsidies) for renewable energy would negatively affect the price of renewable energy stocks, which may transmit to the price of green bond assets. Therefore, policy decisions on the transition of energy to a decarbonized economy should consider the consequences for green bonds, which are also critical for the transition to a climate-resilient economy.

For future work, we would further combine the wavelet correlation and dynamic hedging models to examine the dynamic correlation and volatility spillover between green bonds and renewable energy returns to help hold optimal portfolio weights and hedge ratios especially in times of crisis and under different market conditions. 


\section{Abbreviations:}

COVID-19: The Coronavirus disease 2019

ESDC: The European Sovereign Debt Crisis 2012

S\&P: The Standard \& Poor Co.

VaR: value-at-risk

EU: The European Union

US: The United States

GB: The S\&P Dow Jones Green Bonds Index

RE: renewable energy

ECO: The Wilder Hill Clean Energy Index

GCE: The S\&P 500 Global Clean Index

ERIX: The European Renewable Energy Index

WIND: The ISE Global Wind Energy Index

SOLAR: The MAC Global Solar Equity Index

TECH: The S\&P Renewable Energy and Clean Technology Index

JB: The Jarque-Bera test

Q20: Ljung-Box statistics 20

ARCH-LM: Autoregressive Conditional Heteroskedasticity-Lagrange Multiplier tests ADF: Augmented Dickey and Fuller

PP: Phillips and Perron

KPSS: Kwiatkowski et al. (1992) stationarity test

Corr.: Pearson correlation

CWT: Continuous wavelet transform

DWT: Discrete wavelet transform

VAR: vector autoregressive

AIC: The Akaike information criterion

Acknowledgments: Supports from the "Double-First Class" Think Tank Program of

China University of Mining and Technology (No.2018WHCC01) are acknowledged.

Authors' contributions: Data curation, N.L.; Formal analysis, N.L.; Funding acquisition, N.L. and C.L.; Investigation, N.L. and C.L.; Methodology, N.L.; Resources, N.L. and C.L.; Software, N.L.; Supervision, C.L.; Validation, N.L.; Visualization, N.L.; Writing — original draft, N.L.; Writing — review \& editing, C.L.

Funding: This research was funded by "Double-First Class" Think Tank Program of China University of Mining and Technology (No.2018WHCC01).

\section{Availability of data and materials}

The datasets obtained and analyzed in the current study are available from the corresponding author on reasonable request.

\section{Ethics approval and consent to participate}

Not applicable.

\section{Consent for publication}

All authors agreed to publish the paper. 


\section{Competing interests}

737 The authors declare that they have no competing interests.

\section{Author details}

${ }^{1}$ School of Management, China University of Mining \& Technology, Xuzhou 221116, China.

\section{Reference}

1. Le Quéré, C. ; Jackson, R. B. ; Jones, M. W. ; Smith, A. J. ; Abernethy, S. ; Andrew, R. M. ; De-Gol, A. J. ; Willis, D. R. ; Shan, Y. ; Canade1l, J. G., Temporary reduction in daily global C0 2 emissions during the COVID-19 forced confinement. Nature Climate Change 2020, 1-7.

2. McCollum, D. L. ; Echeverri, L. G. ; Busch, S. ; Pachauri, S. ; Parkinson, S. ; Rogelj, J. ; Krey, V. ; Minx, J. C. ; Nilsson, M. ; Stevance, A. -S., Connecting the sustainable development goals by their energy inter-linkages. Environmental Research Letters 2018, 13, (3), 033006.

3. Xie, F. ; Liu, Y. ; Guan, F.; Wang, N., How to coordinate the relationship between renewable energy consumption and green economic development: from the perspective of technological advancement. Environmental Sciences Europe 2020, 32, (1), 1-15.

4. Brack, W. ; Ait-Aissa, S. ; Backhaus, T. ; Birk, S. ; Barceló, D. ; Burgess, R. ; Cousins, I. ; Dulio, V. ; Escher, B. I. ; Focks, A., Strengthen the European collaborative environmental research to meet European policy goals for achieving a sustainable, non-toxic environment. Environmental Sciences Europe 2019, 31, (1), $1-9$.

5. Ng, T. H. ; Tao, J. Y., Bond financing for renewable energy in Asia. Energy Policy 2016, 95, 509-517.

6. Shishlov, I. ; More1, R. ; Cochran, I., Beyond transparency: unlocking the full potential of green bonds. Institute for Climate Economics 2016, 1-28.

7. Park, S. K., Investors as regulators: Green bonds and the governance challenges of the sustainable finance revolution. Stan. J. Int'1 L. 2018, 54, 1.

8. Azhgaliyeva, D. ; Kapoor, A. ; Liu, Y., Green bonds for financing renewable energy and energy efficiency in South-East Asia: a review of policies. Journal of Sustainable Finance \& Investment 2020, 10, (2), 113-140.

9. Hachenberg, B.; Schiereck, D., Are green bonds priced differently from conventional bonds? Journal of Asset Management 2018, 19, (6), 371-383.

10. Rache1lo, V. THE GREEN BOND MARKET IN EMERGING MARKET ECONOMIES Green Bond Market Development and Green Premium analysis in Emerging Market Economies. Università Ca' Foscari Venezia, 2019.

11. Tang, D. Y.; Zhang, Y., Do shareholders benefit from green bonds? Journal of Corporate Finance 2020, 61, 101427.

12. Naccache, T., Oil price cycles and wavelets. Energy Economics 2011, 33, (2), 338-352.

13. Vacha, L. ; Barunik, J., Co-movement of energy commodities revisited: Evidence from wavelet coherence analysis. Energy Economics 2012, 34, (1), 241-247.

14. Jammazi, R., Cross dynamics of oil-stock interactions: A redundant wavelet analysis. Energy 2012, 44, (1), 750-777. 
15. Reboredo, J. C. ; Rivera-Castro, M. A., Wavelet-based evidence of the impact of

16. Reboredo, J. C. ; Rivera-Castro, M. A., A wavelet decomposition approach to crude oil price and exchange rate dependence. Economic Modelling 2013, 32, 42-57.

17. Madaleno, M. ; Pinho, C., Wavelet dynamics for oil-stock world interactions. Energy Economics 2014, 45, 120-133.

18. Shiller, R. J.; Beltratti, A. E., Stock prices and bond yields: Can their comovements be explained in terms of present value models? Journal of monetary economics 1992, 30, (1), 25-46.

19. Arouri, M. E. H. ; Jouini, J. ; Nguyen, D. K., Volatility spillovers between oil prices and stock sector returns: Implications for portfolio management. Journal of International money and finance 2011, 30, (7), 1387-1405.

20. Bae, K.-H. ; Karolyi, G. A. ; Stulz, R. M., A new approach to measuring financial contagion. The Review of Financial Studies 2003, 16, (3), 717-763.

21. Barsky, R. B. Why don't the prices of stocks and bonds move together?; 0898-2937; National Bureau of Economic Research: 1986.

22. Dean, C. R. ; Young, A. F. ; Meric, I. ; Lee, C. ; Wang, L. ; Sorgenfrei, S. ; Watanabe, K. ; Taniguchi, T. ; Kim, P. ; Shepard, K. L., Boron nitride substrates for highquality graphene electronics. Nature nanotechnology 2010, 5, (10), 722-726.

23. Pham, L., Is it risky to go green? A volatility analysis of the green bond market. Journal of Sustainable Finance \& Investment 2016, 6, (4), 263-291.

24. Bachelet, M. J. ; Becchetti, L. ; Manfredonia, S., The green bonds premium puzzle: The role of issuer characteristics and third-party verification. Sustainability 2019, 11, (4), 1098.

25. Reboredo, J. C., Green bond and financial markets: Co-movement, diversification and price spillover effects. Energy Economics 2018, 74, 38-50.

26. Reboredo, J. C.; Ugolini, A., Price connectedness between green bond and financial markets. Economic Modelling 2019.

27. Reboredo, J. C. ; Ugolini, A. ; Aiube, F. A. L., Network connectedness of green bonds and asset classes. Energy Economics 2020, 86, 104629.

28. Jin, J. ; Han, L. ; Wu, L. ; Zeng, H., The hedging effect of green bonds on carbon market risk. International Review of Financial Analysis 2020, 101509.

29. Nanji, A. ; Calder, A. ; Kolodzie, M., Green Bonds: Fifty Shades of Green. RBC Capital Markets. Available at: http://www. rbc. com/communitysustainability/_assets-custom/pdf/Green-Bonds-Fifty-Shades-of-Green. pdf 2014.

30. Tolliver, C. ; Keeley, A. R. ; Managi, S., Policy targets behind green bonds for renewable energy: Do climate commitments matter? Technological Forecasting and Social Change 2020, 157, 120051.

31. Tao, J. Y., Utilising Green Bonds for Financing Renewable Energy Projects in Developing Asian Countries. Chapters 2016.

32. Liu, N. ; Liu, C. ; Da, B. ; Zhang, T. ; Guan, F., Dependence and risk spillovers between green bonds and clean energy markets. Journal of Cleaner Production 2020, 279, 123595.

33. Hammoudeh, S.; Ajmi, A. N.; Mokni, K., Relationship between green bonds and financial and environmental variables: A novel time-varying causality. Energy Economics 2020, 92, 104941.

34. Nguyen, T. T. H. ; Naeem, M. A. ; Balli, F. ; Balli, H. 0. ; Vo, X. V., Time-frequency comovement among green bonds, stocks, commodities, clean energy, and conventional 
831

832

833

834

835

836

837

838

839

840

841

842

843

844

845

846

847

848

849

850

851

852

853

854

855

856

857

858

859

860

861

862

863

864

865

866

867

868

869

870

871

872

873

874

875

876

877

878

879

bonds. Finance Research Letters 2020, 101739.

35. Le, T.-L.; Abakah, E. J. A. ; Tiwari, A. K., Time and frequency domain connectedness and spill-over among fintech, green bonds and cryptocurrencies in the age of the fourth industrial revolution. Technological Forecasting and Social Change 2020, 162, 120382.

36. Reboredo, J. C., Green bond and financial markets: Co-movement, diversification and price spillover effects. Energy Economics 2018, 74, (AUG.), 38-50.

37. Ugolini; Andrea; Reboredo; Juan; C. ; Rivera-Castro; Miguel; A., Wavelet-based test of co-movement and causality between oil and renewable energy stock prices. Energy Economics 2017.

38. Rezec, M. ; Scholtens, B., Financing energy transformation: The role of renewable energy equity indices. International Journal of Green Energy 2017, 14, (4), 368378.

39. Ahmad, W., On the dynamic dependence and investment performance of crude oil and clean energy stocks. Research in International Business and Finance 2017, 42, 376-389.

40. Bondia, R. ; Ghosh, S. ; Kanjilal, K., International crude oil prices and the stock prices of clean energy and technology companies: evidence from non-linear cointegration tests with unknown structural breaks. Energy 2016, 101, 558-565.

41. Reboredo, J. C., Is there dependence and systemic risk between oil and renewable energy stock prices? Energy Economics 2015, 48, 32-45.

42. Lundgren, A. I. ; Milicevic, A. ; Uddin, G. S. ; Kang, S. H., Connectedness network and dependence structure mechanism in green investments. Energy Economics 2018, $72, \quad 145-153$.

43. Torrence, C. ; Compo, G. P., A practical guide to wavelet analysis. Bulletin of the American Meteorological society 1998, 79, (1), 61-78.

44. Torrence, C. ; Webster, P. J., Interdecadal changes in the ENSO-monsoon system. Journal of climate 1999, 12, (8), 2679-2690.

45. Berry, W. ; Aggarwal, R. ; Inclan, C., Detecting volatility changes across the oil sector. The Journal of Futures Markets (1986-1998) 1996, 47, (1), 313.

46. Abhyankar, A., Does the stock index futures market tend to lead the cash? New evidence from the FT-SE 100 stock index futures market. In Working Paper No. 9601, Department of Accounting and Finance University of Stirling, 1996.

47. Chen, A. -S. ; Wuh Lin, J., Cointegration and detectable linear and nonlinear causality: analysis using the London Metal Exchange lead contract. Applied Economics 2004, 36, (11), 1157-1167.

48. Bekiros, S. D. ; Diks, C. G., The relationship between crude oil spot and futures prices: Cointegration, linear and nonlinear causality. Energy Economics 2008, 30, (5), 2673-2685.

49. Baek, E. G. ; Brock, W. A., A nonparametric test for independence of a multivariate time series. Statistica Sinica 1992, 137-156.

50. Hiemstra, C. ; Jones, J. D., Testing for linear and nonlinear Granger causality in the stock price - volume relation. The Journal of Finance 1994, 49, (5), 16391664.

51. Diks, C. ; Panchenko, V., A new statistic and practical guidelines for nonparametric Granger causality testing. Journal of Economic Dynamics and Control 2006, 30, (9-10), 1647-1669.

52. Akoum, I. ; Graham, M. ; Kivihaho, J. ; Nikkinen, J. ; Omran, M., Co-movement of oil and stock prices in the GCC region: A wavelet analysis. The Quarterly Review of 
908

909

910

911

912

913

Economics and Finance 2012, 52, (4), 385-394.

53. Ranta, M., Contagion among major world markets: a wavelet approach. International Journal of Managerial Finance 2013.

54. Yu, L. ; Li, J.; Tang, L. ; Wang, S., Linear and nonlinear Granger causality investigation between carbon market and crude oil market: A multi-scale approach. Energy Economics 2015, 51, 300-311. 
915 Fig.1. Time-series plot of the green bonds and renewable energy pairs. Note: The left axis

916 represents the green bond index price level. The right axis represents the renewable energy

917 price level.

918

919

920

921

922

GB
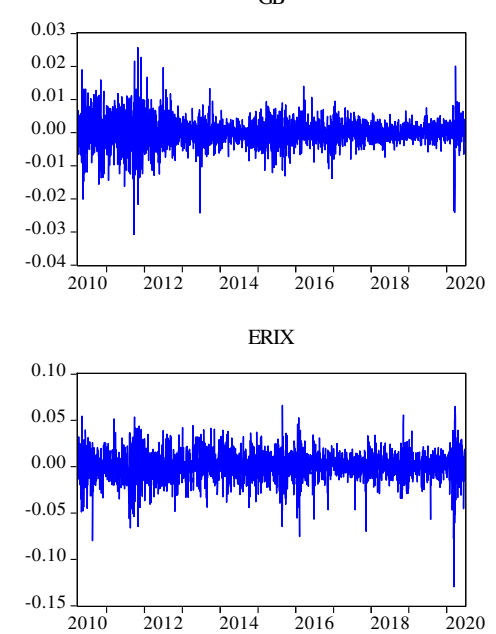

ECO
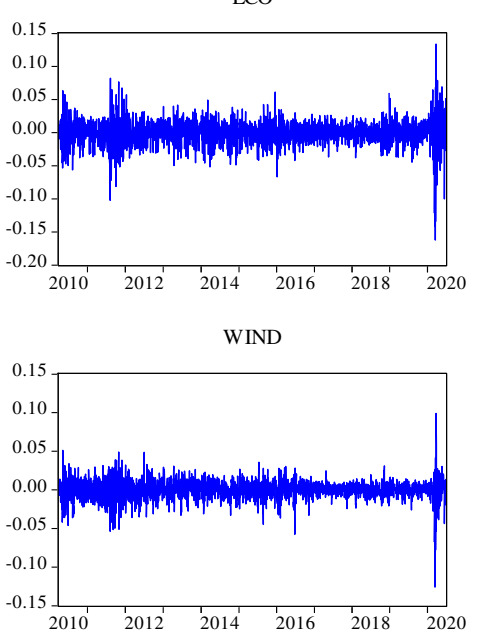

GCE

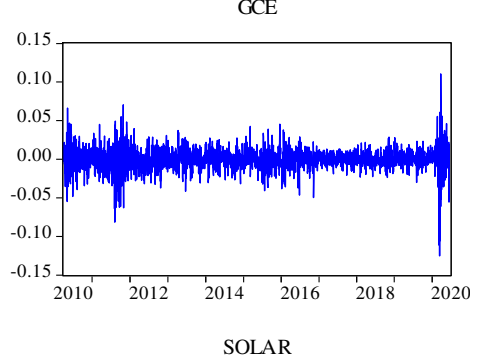

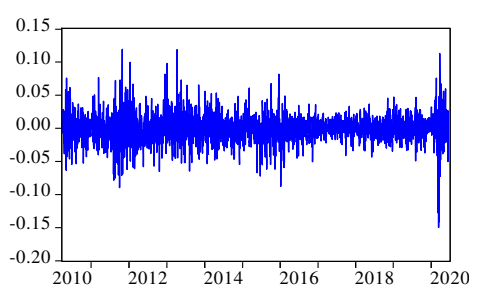

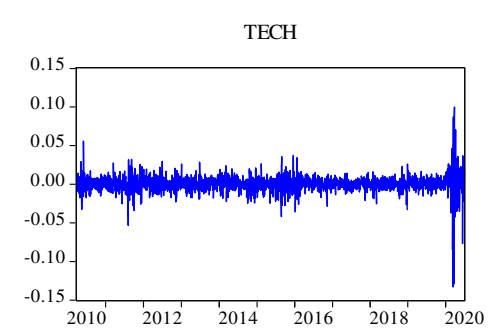


949

950

951

952

953

954

955

956

957

958

959

960

961

962

963

964

965

966

967

968

969

970

971

972

973

974

975

976

977

Out-of-Phase



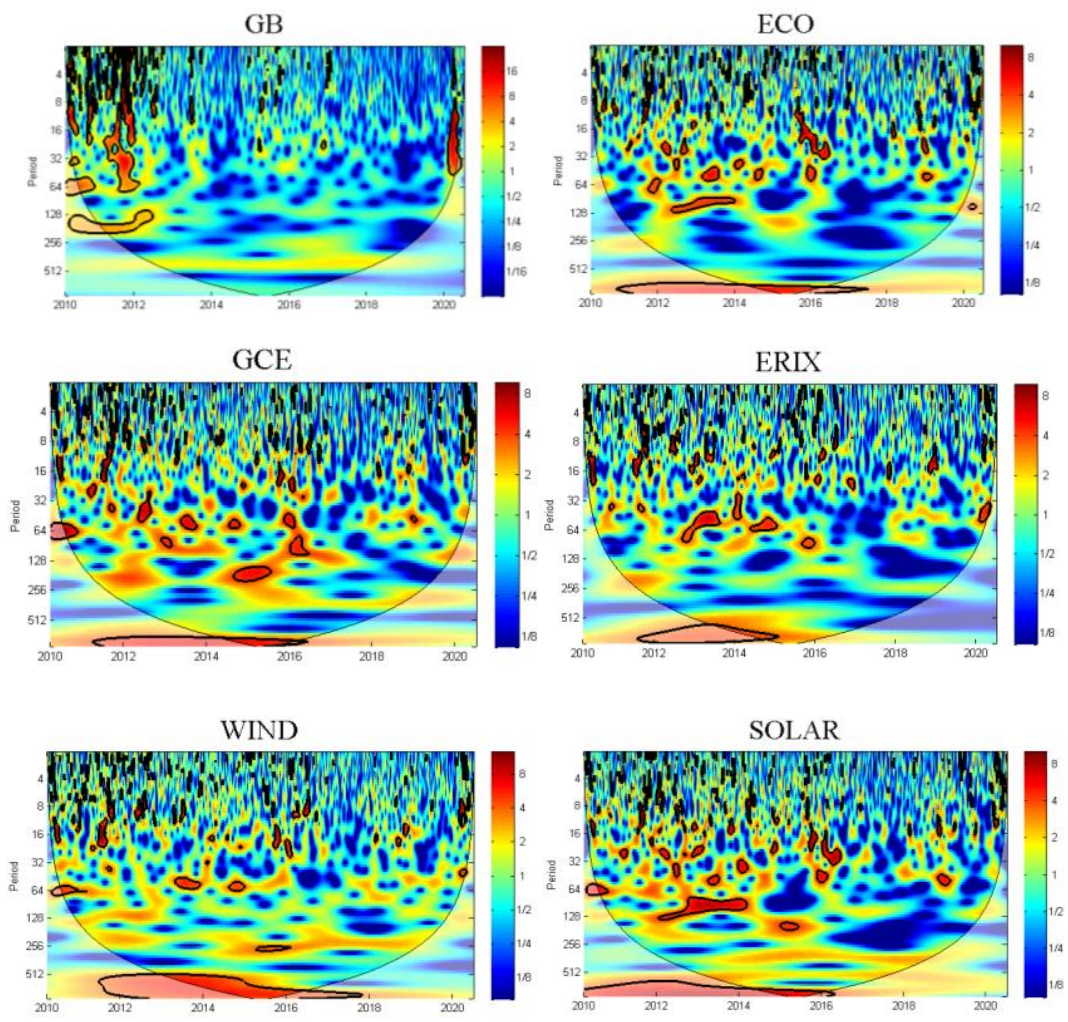

TECH

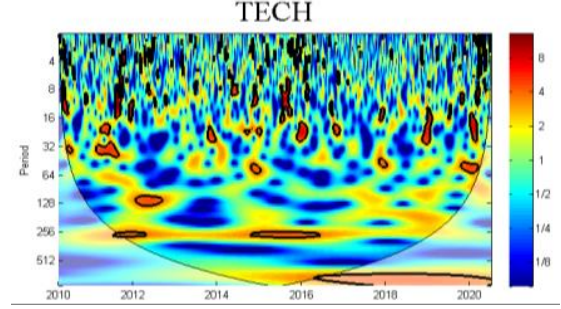


1012 Fig.4. Continuous wavelet transforms for the green bonds and six renewable energy 1013 markets. Note: The dark red (blue) indicates strong (smooth) fluctuations and the bold 1014 black outline indicates the wavelet power spectrum generated from the Monte Carlo 1015 simulation of the 5\% significance level. The region affected by the edge effect is 1016 represented by the black curve and defines the cone of influence. The horizontal axis 1017 indicates time (year) and the vertical axis indicates period (day).

1018

1019

1020

1021

1022

1023

1024

1025

1026

1027

1028

1029

1030

1031

1032

1033

1034

1035

1036

1037

1038

1039

1040

1041

1042
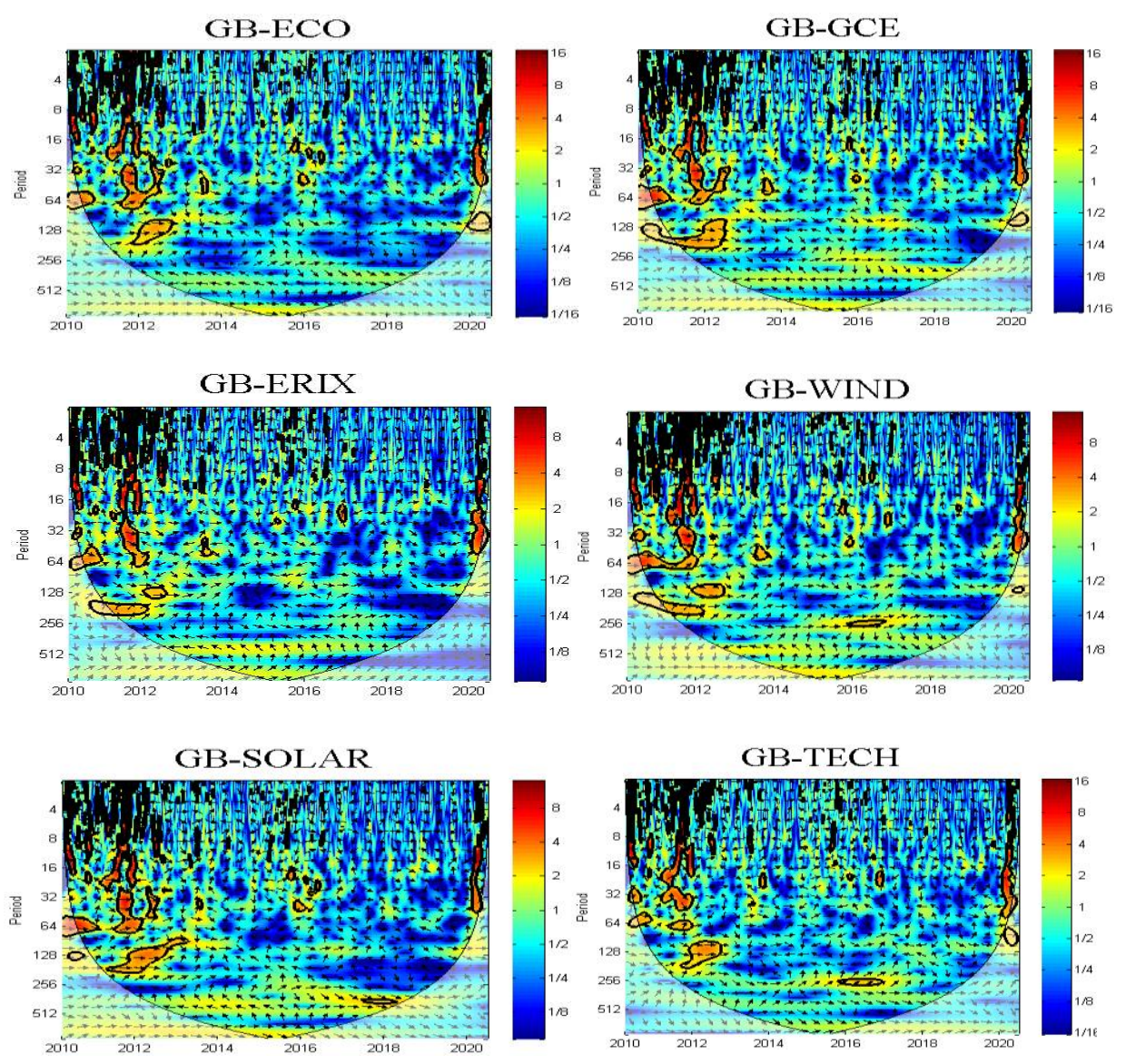
1045 Fig. 5. Cross-wavelet transforms for green bonds and renewable energy indices. Note: the 1046 horizontal axis presents time and the vertical axis shows frequency (days). The warmer 1047 color of the region, the higher the dependence between the pairs.

1048

1049

1050

1051

1052

1053

1054

1055

1056

1057

1058

1059

1060

1061

1062

1063

1064

1065

1066

1067

1068

1069

1070

1071

1072

1073

1074
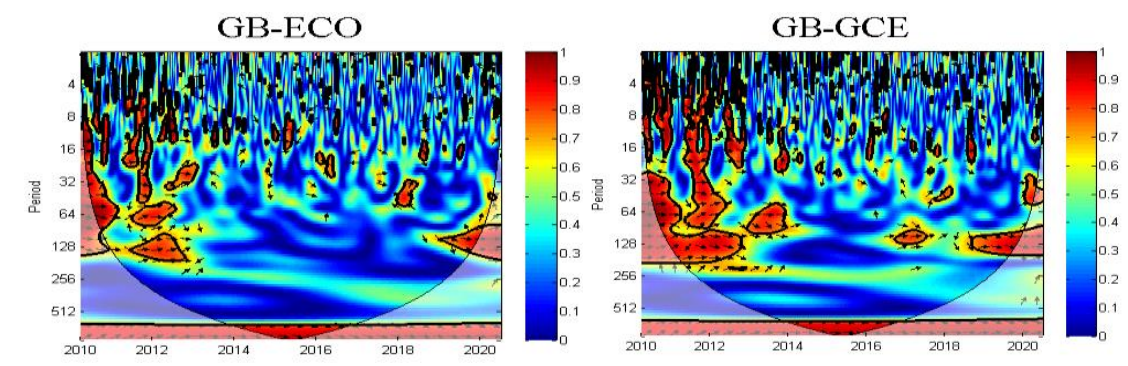

GB-ERIX
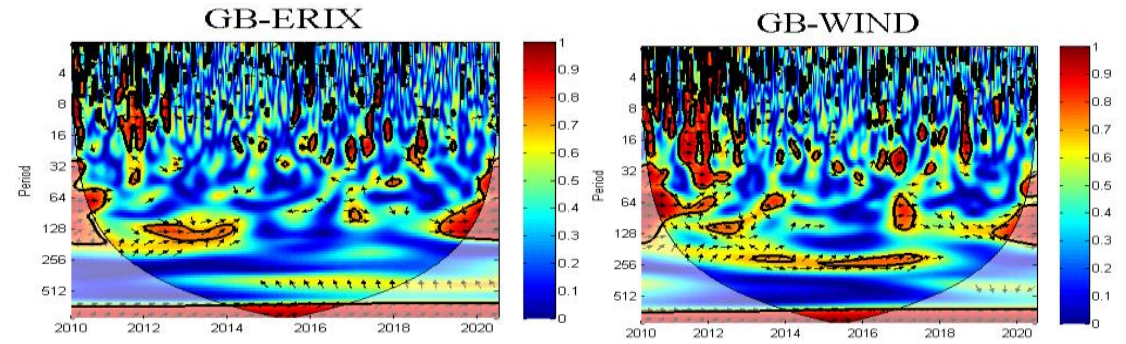

GB-SOLAR

GB-TECH
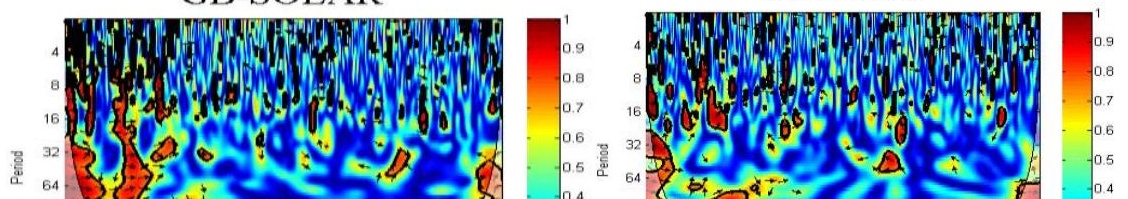
Fig.6. Wavelet coherence of green bonds and renewable energy pairs. Note: refer to Fig.4.

Table1. Descriptive statistics for green bonds and renewable energy return series.

\begin{tabular}{cccccccc}
\hline & GB & ECO & GCE & ERIX & WIND & SOLAR & TECH \\
\hline Mean & 0.0001 & -0.0001 & -0.0001 & 0.0002 & 0.0001 & -0.0003 & 0.0002 \\
Maximum & 0.026 & 0.134 & 0.110 & 0.066 & 0.099 & 0.120 & 0.100 \\
Minimum & -0.031 & -0.162 & -0.125 & -0.130 & -0.126 & -0.150 & -0.133 \\
Std. Dev. & 0.004 & 0.018 & 0.014 & 0.015 & 0.012 & 0.021 & 0.011 \\
Skewness & -0.31 & -0.75 & -0.75 & -0.54 & -0.72 & -0.19 & -1.46 \\
Kurtosis & 8.43 & 11.23 & 12.29 & 7.19 & 12.37 & 7.30 & 33.99 \\
JB & 3321.7 & 7793.4 & 9843.3 & 2081.0 & 9996.7 & 2072.9 & 107807.4 \\
ADF & -51.40 & -32.96 & -18.13 & -49.55 & -32.74 & -44.30 & -19.33 \\
PP & -51.41 & -51.18 & -46.60 & -49.54 & -46.92 & -44.41 & -54.09 \\
KPSS & 0.11 & 0.30 & 0.32 & 0.43 & 0.22 & 0.30 & 0.04 \\
Q (20) & $30.36 *$ & $85.86 * * *$ & $118.58 * * *$ & $32.64 * *$ & $76.98 * * *$ & $109.39 * * *$ & $116.08 * * *$ \\
ARCH- & $31.17 * * *$ & $37.23 * * *$ & $48.93 * * *$ & $24.77 * * *$ & $31.99 * * *$ & $29.98 * * *$ & $29.14 * * *$ \\
LM (5) & & & & & & & 0.26 \\
Corr. & 1.00 & 0.22 & 0.37 & 0.26 & 0.50 & 0.26 & 0.15 \\
\hline
\end{tabular}

1089 Note: Daily data between March 29, 2010 and June 30, 2020. Notes. JB is used to test for 1090 normality Jarque-Bera $\chi 2$ statistic. Q (20) denotes the Ljung-Box statistics for serial returns 1091 computed with 20 lags. ARCH-LM (5) is Engle's heteroscedasticity LM test, calculated 1092 using 5 lags. ADF, PP, and KPSS stand for Augmented Dickey and Fuller (1979) and 1093 Phillips and Perron (1988) unit root test and Kwiatkowski et al. (1992) stationarity test, 1094 respectively. Corr. is the Pearson correlation for each renewable energy index with green 1095 bonds. As usual, ***, **and * denote significance at 1\%, 5\% and 10\%, respectively. 


\begin{tabular}{|c|c|c|c|c|c|c|}
\hline \multirow[t]{2}{*}{ Variables } & \multirow[t]{2}{*}{ Lags } & \multicolumn{2}{|c|}{$\begin{array}{l}\text { H0: GB does not } \\
\text { cause RE }\end{array}$} & \multicolumn{2}{|c|}{$\begin{array}{l}\text { H0: RE does not } \\
\text { cause GB }\end{array}$} & \multirow[t]{2}{*}{ Results } \\
\hline & & F-test & P-Value & F-test & P-Value & \\
\hline GB \& ECO & 7 & 1.925 & 0.0618 & 9.494 & 0.000 & $\mathrm{~GB} \leftarrow \mathrm{ECO}$ \\
\hline GB \& GCE & 10 & 2.571 & 0.004 & 5.762 & 0.000 & $\mathrm{~GB} \Leftrightarrow \mathrm{GCE}$ \\
\hline GB \& ERIX & 5 & 3.021 & 0.010 & 2.996 & 0.011 & $\mathrm{~GB} \Leftrightarrow \mathrm{ERIX}$ \\
\hline GB \& WIND & 10 & 2.391 & 0.008 & 3.233 & 0.000 & $\mathrm{~GB} \Leftrightarrow \mathrm{WIND}$ \\
\hline GB \& SOLAR & 7 & 1.803 & 0.083 & 7.086 & 0.000 & $\mathrm{~GB} \leftarrow \mathrm{SOLAR}$ \\
\hline GB \& TECH & 10 & 3.539 & 0.000 & 4.133 & 0.000 & $\mathrm{~GB} \Leftrightarrow \mathrm{TECH}$ \\
\hline
\end{tabular}

1112 Note: GB refer to green bonds. RE stands for renewable energy. The lag number is 1113 determined based on the AIC criterion.

1114 (Source: Authors' calculation.)

1115 


\begin{tabular}{|c|c|c|c|c|c|c|}
\hline \multirow[t]{2}{*}{ Variables } & \multirow[t]{2}{*}{ Lags } & \multicolumn{2}{|c|}{$\begin{array}{c}\text { H0: GB does not } \\
\text { cause RE }\end{array}$} & \multicolumn{2}{|c|}{$\begin{array}{c}\text { H0: } \text { RE does not } \\
\text { cause GB }\end{array}$} & \multirow[t]{2}{*}{ Results } \\
\hline & & F-test & P-Value & F-test & P-Value & \\
\hline \multicolumn{7}{|l|}{ Panel A: D1 } \\
\hline GB \& ECO & 10 & 1.158 & 0.315 & 2.369 & 0.009 & $\mathrm{~GB} \leftarrow \mathrm{ECO}$ \\
\hline GB \& GCE & 10 & 1.730 & 0.069 & 2.419 & 0.007 & $\mathrm{~GB} \leftarrow \mathrm{GCE}$ \\
\hline GB \& ERIX & 10 & 2.171 & 0.017 & 1.674 & 0.081 & $\mathrm{~GB} \Leftrightarrow \mathrm{ERIX}$ \\
\hline GB \& WIND & 10 & 0.935 & 0.499 & 2.002 & 0.030 & GB $\leftarrow W I N D$ \\
\hline GB \& SOLAR & 10 & 0.657 & 0.765 & 2.507 & 0.005 & $\mathrm{~GB} \leftarrow \mathrm{SOLAR}$ \\
\hline GB \& TECH & 10 & 1.515 & 0.128 & 1.072 & 0.381 & No causality \\
\hline \multicolumn{7}{|l|}{ Panel B: D2 } \\
\hline GB \& ECO & 10 & 3.468 & 0.000 & 3.015 & 0.001 & $\mathrm{~GB} \Leftrightarrow \mathrm{ECO}$ \\
\hline GB \& GCE & 10 & 2.078 & 0.023 & 2.050 & 0.025 & $\mathrm{~GB} \Leftrightarrow \mathrm{GCE}$ \\
\hline GB \& ERIX & 10 & 1.008 & 0.434 & 1.596 & 0.101 & No causality \\
\hline GB \& WIND & 10 & 2.034 & 0.027 & 1.854 & 0.047 & GB $\Leftrightarrow$ WIND \\
\hline GB \& SOLAR & 10 & 1.955 & 0.034 & 1.330 & 0.208 & $\mathrm{~GB} \rightarrow \mathrm{SOLAR}$ \\
\hline GB \& TECH & 10 & 4.104 & 0.000 & 4.050 & 0.000 & $\mathrm{~GB} \Leftrightarrow \mathrm{TECH}$ \\
\hline \multicolumn{7}{|l|}{ Panel C: D3 } \\
\hline GB \& ECO & 10 & 5.669 & 0.000 & 6.263 & 0.000 & $\mathrm{~GB} \Leftrightarrow \mathrm{ECO}$ \\
\hline GB \& GCE & 10 & 7.379 & 0.000 & 6.717 & 0.000 & $\mathrm{~GB} \Leftrightarrow \mathrm{GCE}$ \\
\hline GB \& ERIX & 10 & 6.399 & 0.000 & 7.038 & 0.000 & $\mathrm{~GB} \Leftrightarrow \mathrm{ERIX}$ \\
\hline
\end{tabular}




\begin{tabular}{|c|c|c|c|c|c|c|}
\hline GB \& WIND & 10 & 7.091 & 0.000 & 6.726 & 0.000 & GB $\Leftrightarrow$ WIND \\
\hline GB \& SOLAR & 10 & 4.387 & 0.000 & 4.570 & 0.000 & $\mathrm{~GB} \Leftrightarrow \mathrm{SOLAR}$ \\
\hline GB \& TECH & 10 & 5.105 & 0.000 & 4.926 & 0.000 & $\mathrm{~GB} \Leftrightarrow \mathrm{TECH}$ \\
\hline \multicolumn{7}{|l|}{ Panel D: D4 } \\
\hline GB \& ECO & 10 & 5.717 & 0.000 & 3.653 & 0.000 & $\mathrm{~GB} \Leftrightarrow \mathrm{ECO}$ \\
\hline $\mathrm{GB} \& \mathrm{GCE}$ & 10 & 3.804 & 0.000 & 2.805 & 0.002 & $\mathrm{~GB} \Leftrightarrow \mathrm{GCE}$ \\
\hline GB \& ERIX & 10 & 5.135 & 0.000 & 2.732 & 0.002 & $\mathrm{~GB} \Leftrightarrow \mathrm{ERIX}$ \\
\hline GB \& WIND & 10 & 4.368 & 0.000 & 1.395 & 0.176 & $\mathrm{~GB} \rightarrow \mathrm{WIND}$ \\
\hline GB \& SOLAR & 10 & 3.894 & 0.000 & 3.066 & 0.001 & $\mathrm{~GB} \Leftrightarrow \mathrm{SOLAR}$ \\
\hline GB \& TECH & 10 & 2.840 & 0.002 & 3.113 & 0.001 & $\mathrm{~GB} \Leftrightarrow \mathrm{TECH}$ \\
\hline \multicolumn{7}{|l|}{ Panel E: D5 } \\
\hline GB \& ECO & 10 & 0.323 & 0.975 & 0.816 & 0.613 & No causality \\
\hline GB \& GCE & 10 & 1.156 & 0.316 & 5.164 & 0.000 & $\mathrm{~GB} \leftarrow \mathrm{GCE}$ \\
\hline GB \& ERIX & 10 & 2.839 & 0.002 & 3.041 & 0.001 & $\mathrm{~GB} \Leftrightarrow \mathrm{ERIX}$ \\
\hline GB \& WIND & 10 & 2.477 & 0.006 & 3.590 & 0.000 & $\mathrm{~GB} \Leftrightarrow \mathrm{WIND}$ \\
\hline GB \& SOLAR & 10 & 0.634 & 0.786 & 1.787 & 0.058 & No causality \\
\hline $\mathrm{GB} \& \mathrm{TECH}$ & 10 & 0.678 & 0.746 & 0.467 & 0.912 & No causality \\
\hline \multicolumn{7}{|l|}{ Panel F: D6 } \\
\hline $\mathrm{GB} \& \mathrm{ECO}$ & 10 & 2.694 & 0.003 & 0.807 & 0.622 & $\mathrm{~GB} \rightarrow \mathrm{ECO}$ \\
\hline GB \& GCE & 10 & 4.253 & 0.000 & 0.448 & 0.923 & $\mathrm{~GB} \rightarrow \mathrm{GCE}$ \\
\hline GB \& ERIX & 10 & 5.309 & 0.000 & 0.296 & 0.982 & $\mathrm{~GB} \rightarrow$ ERIX \\
\hline GB \& WIND & 10 & 2.847 & 0.002 & 0.463 & 0.914 & GB $\rightarrow$ WIND \\
\hline GB \& SOLAR & 10 & 5.671 & 0.000 & 0.578 & 0.833 & $\mathrm{~GB} \rightarrow$ SOLAR \\
\hline GB \& TECH & 10 & 2.328 & 0.010 & 1.288 & 0.231 & $\mathrm{~GB} \rightarrow \mathrm{TECH}$ \\
\hline \multicolumn{7}{|l|}{ Panel G: D7 } \\
\hline $\mathrm{GB} \& \mathrm{ECO}$ & 10 & 0.124 & 1.000 & -0.279 & 1.000 & No causality \\
\hline GB \& GCE & 10 & -0.128 & 1.000 & 0.216 & 0.995 & No causality \\
\hline GB \& ERIX & 10 & 14.372 & 0.000 & 12.175 & 0.000 & $\mathrm{~GB} \Leftrightarrow \mathrm{ERIX}$ \\
\hline GB \& WIND & 10 & 4.086 & 0.000 & 2.673 & 0.003 & $\mathrm{~GB} \Leftrightarrow \mathrm{WIND}$ \\
\hline GB \& SOLAR & 10 & 0.825 & 0.605 & 1.625 & 0.093 & No causality \\
\hline GB \& TECH & 10 & 1.614 & 0.096 & 1.805 & 0.055 & No causality \\
\hline \multicolumn{7}{|l|}{ Panel H: D8 } \\
\hline $\mathrm{GB} \& \mathrm{ECO}$ & 3 & 11.815 & 0.000 & 5.804 & 0.001 & $\mathrm{~GB} \Leftrightarrow \mathrm{ECO}$ \\
\hline $\mathrm{GB} \& \mathrm{GCE}$ & 3 & 9.978 & 0.000 & 12.695 & 0.000 & $\mathrm{~GB} \Leftrightarrow \mathrm{GCE}$ \\
\hline GB \& ERIX & 3 & 9.094 & 0.000 & 10.126 & 0.000 & $\mathrm{~GB} \Leftrightarrow \mathrm{ERIX}$ \\
\hline GB \& WIND & 3 & 14.630 & 0.000 & 22.698 & 0.000 & GB $\Leftrightarrow$ WIND \\
\hline GB \& SOLAR & 3 & 3.075 & 0.027 & 14.145 & 0.000 & $\mathrm{~GB} \Leftrightarrow \mathrm{SOLAR}$ \\
\hline GB \& TECH & 3 & 113.929 & 0.000 & 24.713 & 0.000 & $\mathrm{~GB} \Leftrightarrow \mathrm{TECH}$ \\
\hline
\end{tabular}

1138 Note:(refer to table 2.) 

Table 4 Non-linear Granger causality test on returns

\begin{tabular}{|c|c|c|c|c|c|}
\hline \multirow{3}{*}{ Variables } & \multicolumn{2}{|c|}{ H0: GB does not } & \multicolumn{2}{|c|}{ H0: RE does not cause } & \multirow{3}{*}{ Results } \\
\hline & \multicolumn{2}{|c|}{ cause $\mathrm{RE}$} & \multicolumn{2}{|c|}{ GB } & \\
\hline & T-test & P-Value & T-test & P-Value & \\
\hline GB \& ECO & 3.333 & 0.000 & 4.069 & 0.000 & $\mathrm{~GB} \Leftrightarrow \mathrm{ECO}$ \\
\hline GB \& GCE & 4.970 & 0.000 & 4.438 & 0.000 & $\mathrm{~GB} \Leftrightarrow \mathrm{GCE}$ \\
\hline GB \& ERIX & 3.265 & 0.001 & 2.588 & 0.005 & $\mathrm{~GB} \Leftrightarrow \mathrm{ERIX}$ \\
\hline GB \& WIND & 4.830 & 0.000 & 3.994 & 0.000 & GB $\Leftrightarrow$ WIND \\
\hline GB \& SOLAR & 4.472 & 0.000 & 4.301 & 0.000 & $\mathrm{~GB} \Leftrightarrow \mathrm{SOLAR}$ \\
\hline $\mathrm{GB} \& \mathrm{TECH}$ & 2.818 & 0.002 & 2.541 & 0.006 & $\mathrm{~GB} \Leftrightarrow \mathrm{TECH}$ \\
\hline
\end{tabular}

1165 Note:(refer to table 2.) 


\begin{tabular}{lccccl}
\hline \multirow{2}{*}{ Time scale } & \multicolumn{2}{c}{ H0: GB does not cause } & \multicolumn{2}{c}{ H0: RE does not cause } & \\
& \multicolumn{2}{c}{ RE } & \multicolumn{2}{c}{ GB } & Results \\
\cline { 2 - 4 } & T-test & P-Value & T-test & P-Value & \\
\hline Panel A: D1 & & & & & \\
GB \& ECO & 3.059 & 0.001 & 3.627 & 0.000 & GB $\Leftrightarrow$ ECO \\
GB \& GCE & 4.634 & 0.000 & 4.386 & 0.000 & GB $\Leftrightarrow$ GCE \\
GB \& ERIX & 2.514 & 0.006 & 2.179 & 0.015 & GB $\Leftrightarrow$ ERIX \\
GB \& WIND & 4.521 & 0.000 & 5.039 & 0.000 & GB $\Leftrightarrow$ WIND \\
GB \& SOLAR & 4.246 & 0.000 & 4.218 & 0.000 & GB $\Leftrightarrow$ SOLAR \\
GB \& TECH & 2.145 & 0.016 & 2.716 & 0.003 & GB $\Leftrightarrow$ TECH \\
Panel B: D2 & & & & & \\
\hline
\end{tabular}




\begin{tabular}{|c|c|c|c|c|c|}
\hline $\mathrm{GB} \& \mathrm{ECO}$ & 2.785 & 0.003 & 2.052 & 0.020 & $\mathrm{~GB} \Leftrightarrow \mathrm{ECO}$ \\
\hline GB \& GCE & 4.847 & 0.000 & 3.282 & 0.001 & $\mathrm{~GB} \Leftrightarrow \mathrm{GCE}$ \\
\hline GB \& ERIX & 3.716 & 0.000 & 2.104 & 0.018 & $\mathrm{~GB} \Leftrightarrow \mathrm{ERIX}$ \\
\hline GB \& WIND & 5.538 & 0.000 & 3.950 & 0.000 & $\mathrm{~GB} \Leftrightarrow \mathrm{WIND}$ \\
\hline GB \& SOLAR & 3.615 & 0.000 & 2.641 & 0.004 & $\mathrm{~GB} \Leftrightarrow \mathrm{SOLAR}$ \\
\hline GB \& TECH & 2.039 & 0.021 & 2.151 & 0.016 & $\mathrm{~GB} \Leftrightarrow \mathrm{TECH}$ \\
\hline \multicolumn{6}{|l|}{ Panel C: D3 } \\
\hline GB \& ECO & 5.046 & 0.000 & 3.315 & 0.000 & $\mathrm{~GB} \Leftrightarrow \mathrm{ECO}$ \\
\hline GB \& GCE & 6.579 & 0.000 & 6.278 & 0.000 & $\mathrm{~GB} \Leftrightarrow \mathrm{GCE}$ \\
\hline GB \& ERIX & 5.553 & 0.000 & 4.814 & 0.000 & $\mathrm{~GB} \Leftrightarrow \mathrm{ERIX}$ \\
\hline GB \& WIND & 6.000 & 0.000 & 6.945 & 0.000 & $\mathrm{~GB} \Leftrightarrow \mathrm{WIND}$ \\
\hline GB \& SOLAR & 4.451 & 0.000 & 3.466 & 0.000 & $\mathrm{~GB} \Leftrightarrow \mathrm{SOLAR}$ \\
\hline GB \& TECH & 4.072 & 0.000 & 3.061 & 0.001 & $\mathrm{~GB} \Leftrightarrow \mathrm{TECH}$ \\
\hline \multicolumn{6}{|l|}{ Panel D: D4 } \\
\hline GB \& ECO & 3.366 & 0.000 & 1.711 & 0.044 & $\mathrm{~GB} \Leftrightarrow \mathrm{ECO}$ \\
\hline GB \& GCE & 4.173 & 0.000 & 3.521 & 0.000 & $\mathrm{~GB} \Leftrightarrow \mathrm{GCE}$ \\
\hline GB \& ERIX & 2.600 & 0.005 & 1.104 & 0.135 & GB $\rightarrow$ ERIX \\
\hline GB \& WIND & 3.911 & 0.000 & 3.245 & 0.001 & GB $\Leftrightarrow$ WIND \\
\hline GB \& SOLAR & 2.996 & 0.001 & 0.796 & 0.213 & $\mathrm{~GB} \rightarrow$ SOLAR \\
\hline GB \& TECH & 2.929 & 0.002 & 2.442 & 0.007 & $\mathrm{~GB} \Leftrightarrow \mathrm{TECH}$ \\
\hline \multicolumn{6}{|l|}{ Panel E: D5 } \\
\hline GB \& ECO & 2.411 & 0.008 & 2.387 & 0.009 & $\mathrm{~GB} \Leftrightarrow \mathrm{ECO}$ \\
\hline GB \& GCE & 3.180 & 0.001 & 2.508 & 0.006 & $\mathrm{~GB} \Leftrightarrow \mathrm{GCE}$ \\
\hline GB \& ERIX & 2.292 & 0.011 & 1.668 & 0.048 & $\mathrm{~GB} \Leftrightarrow \mathrm{ERIX}$ \\
\hline GB \& WIND & 3.689 & 0.000 & 2.216 & 0.013 & GB $\Leftrightarrow$ WIND \\
\hline GB \& SOLAR & 2.567 & 0.005 & 2.241 & 0.013 & $\mathrm{~GB} \Leftrightarrow \mathrm{SOLAR}$ \\
\hline GB \& TECH & 0.583 & 0.280 & 2.497 & 0.006 & $\mathrm{~GB} \leftarrow \mathrm{TECH}$ \\
\hline \multicolumn{6}{|l|}{ Panel F: D6 } \\
\hline GB \& ECO & 3.510 & 0.000 & 1.709 & 0.044 & $\mathrm{~GB} \Leftrightarrow \mathrm{ECO}$ \\
\hline GB \& GCE & 4.016 & 0.000 & 3.725 & 0.000 & $\mathrm{~GB} \Leftrightarrow \mathrm{GCE}$ \\
\hline GB \& ERIX & 2.935 & 0.002 & 2.173 & 0.015 & $\mathrm{~GB} \Leftrightarrow \mathrm{ERIX}$ \\
\hline GB \& WIND & 4.474 & 0.000 & 4.062 & 0.000 & GB $\Leftrightarrow$ WIND \\
\hline GB \& SOLAR & 3.680 & 0.000 & 3.083 & 0.001 & $\mathrm{~GB} \Leftrightarrow \mathrm{SOLAR}$ \\
\hline GB \& TECH & 2.550 & 0.005 & 2.569 & 0.005 & $\mathrm{~GB} \Leftrightarrow \mathrm{TECH}$ \\
\hline \multicolumn{6}{|l|}{ Panel G: D7 } \\
\hline $\mathrm{GB} \& \mathrm{ECO}$ & -2.456 & 0.993 & -2.661 & 0.996 & No causality \\
\hline GB \& GCE & 1.987 & 0.023 & 2.969 & 0.001 & $\mathrm{~GB} \Leftrightarrow \mathrm{GCE}$ \\
\hline GB \& ERIX & -3.423 & 1.000 & 3.413 & 0.000 & GB $\leftarrow$ ERIX \\
\hline GB \& WIND & 1.652 & 0.049 & 3.568 & 0.000 & GB $\Leftrightarrow$ WIND \\
\hline GB \& SOLAR & 0.070 & 0.472 & 0.761 & 0.223 & No causality \\
\hline GB \& TECH & -3.249 & 0.999 & -1.352 & 0.912 & No causality \\
\hline \multicolumn{6}{|l|}{ Panel H: D8 } \\
\hline GB \& ECO & -0.338 & 0.632 & 2.226 & 0.013 & $\mathrm{~GB} \leftarrow \mathrm{ECO}$ \\
\hline GB \& GCE & 1.860 & 0.031 & 0.413 & 0.340 & $\mathrm{~GB} \rightarrow \mathrm{GCE}$ \\
\hline
\end{tabular}




\begin{tabular}{llllll}
\hline GB \& ERIX & 0.618 & 0.268 & -2.373 & 0.991 & No causality \\
GB \& WIND & 2.126 & 0.017 & -2.603 & 0.995 & GB $\rightarrow$ WIND \\
GB \& SOLAR & 1.587 & 0.056 & 0.988 & 0.162 & No causality \\
GB \& TECH & 2.473 & 0.007 & 4.242 & 0.000 & GB $\Leftrightarrow$ TECH \\
\hline
\end{tabular}

1193 Note:(refer to table 2.) 
Figures
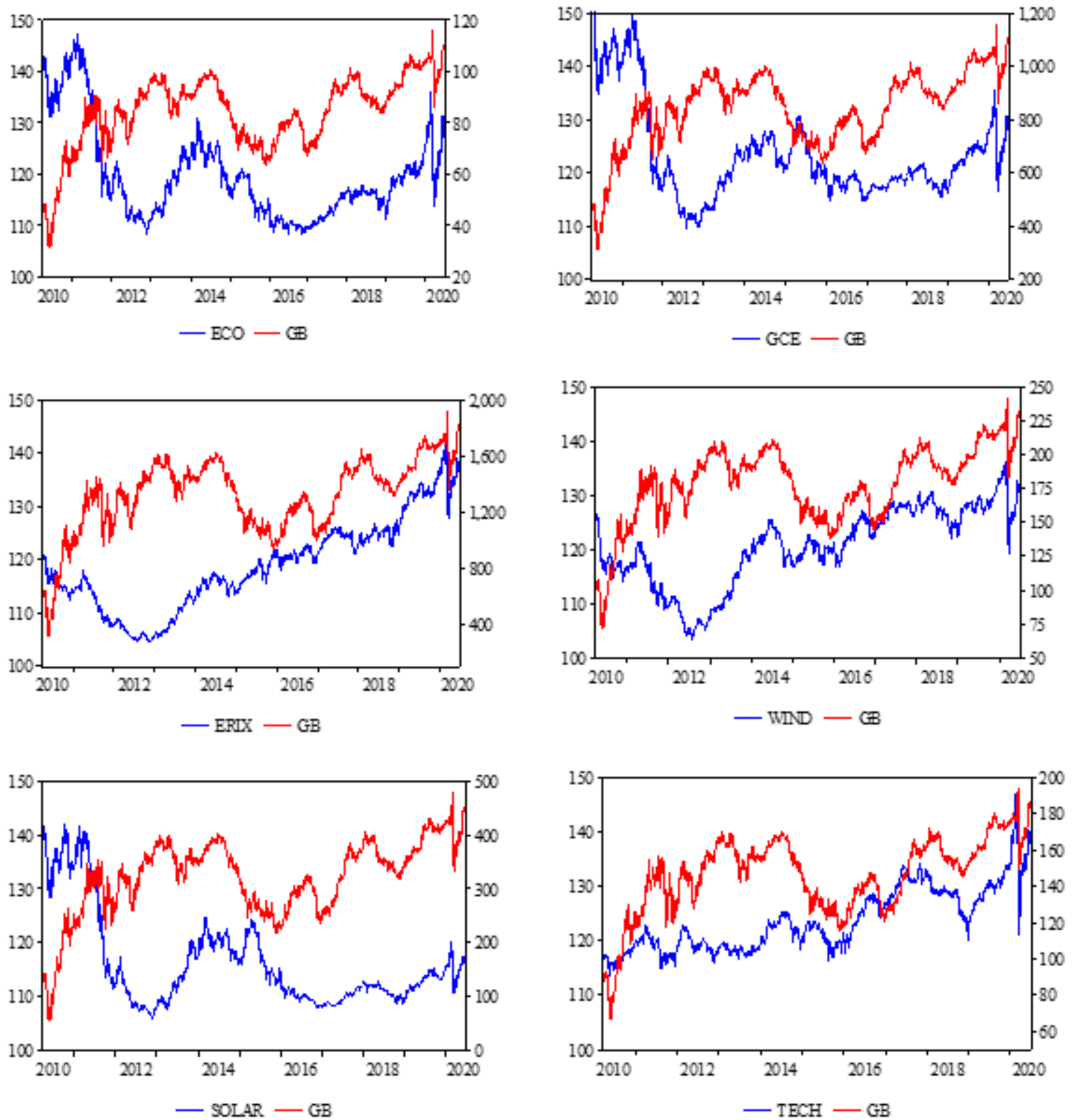

Figure 1

Time-series plot of the green bonds and renewable energy pairs. Note: The left axis represents the green bond index price level. The right axis represents the renewable energy price level. 

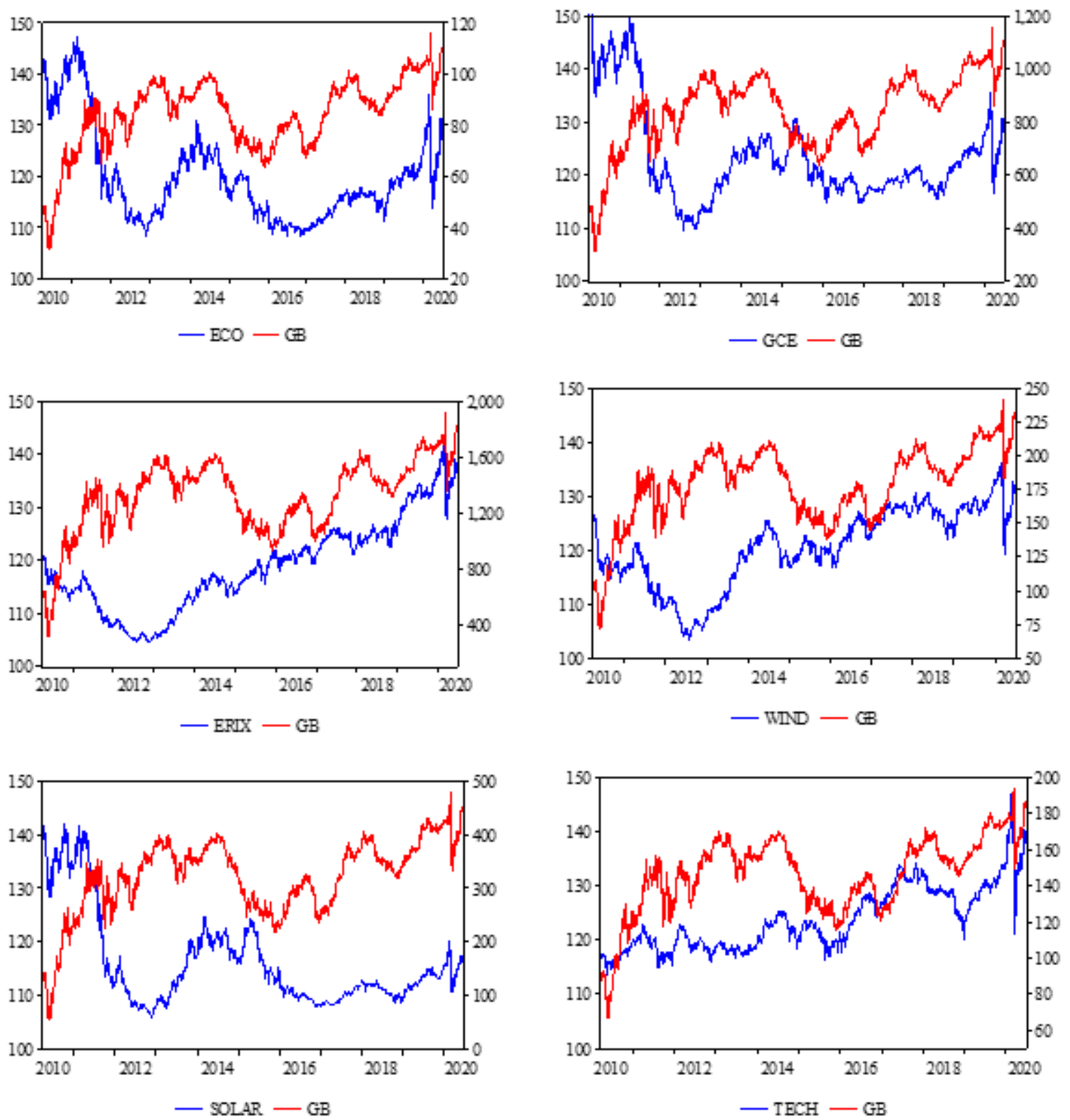

Figure 1

Time-series plot of the green bonds and renewable energy pairs. Note: The left axis represents the green bond index price level. The right axis represents the renewable energy price level. 
GB

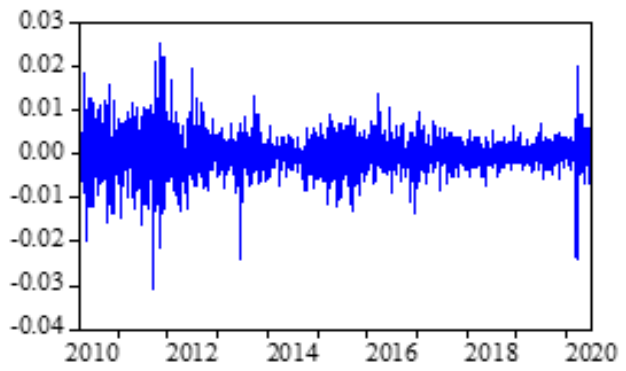

ERIX

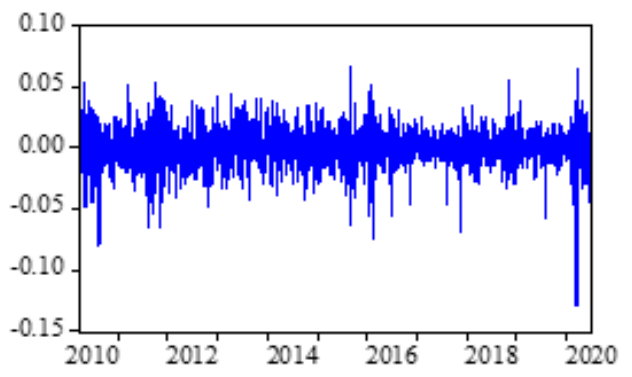

TECH

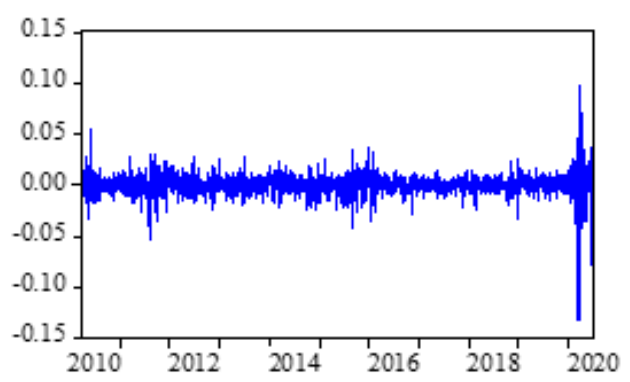

ECO

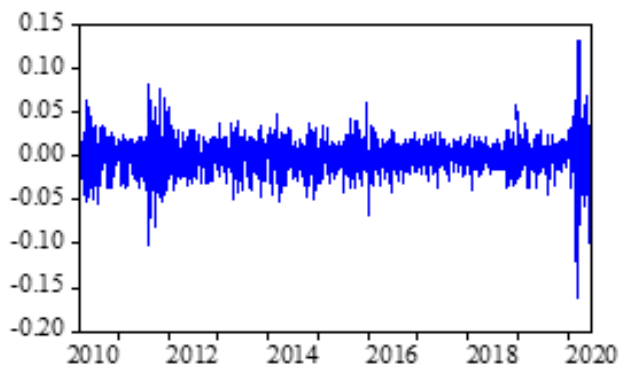

WIND

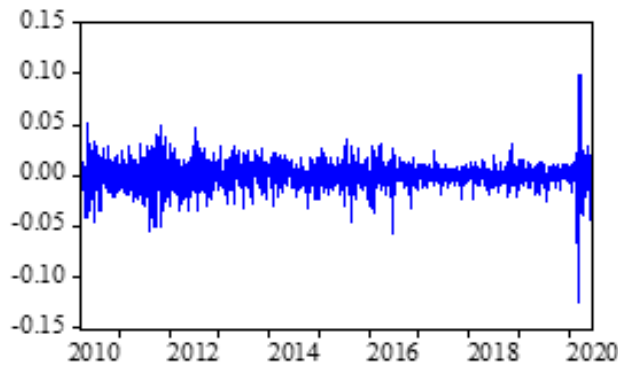

GCE

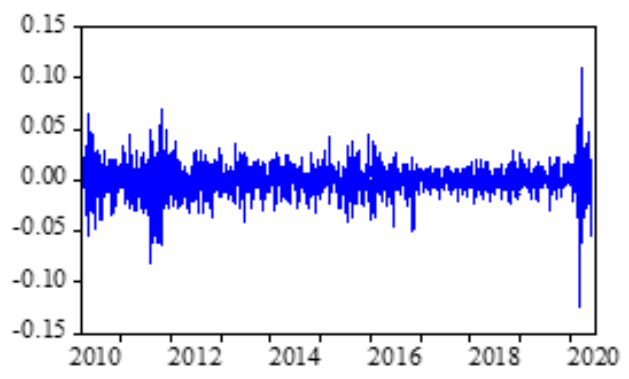

SOLAR

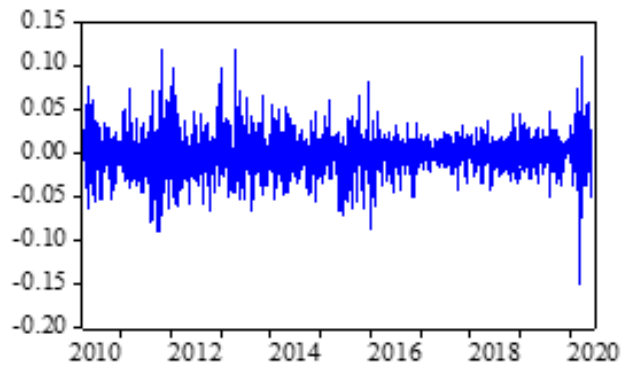

Figure 2

Time-series plot of green bonds and renewable energy returns. 
GB

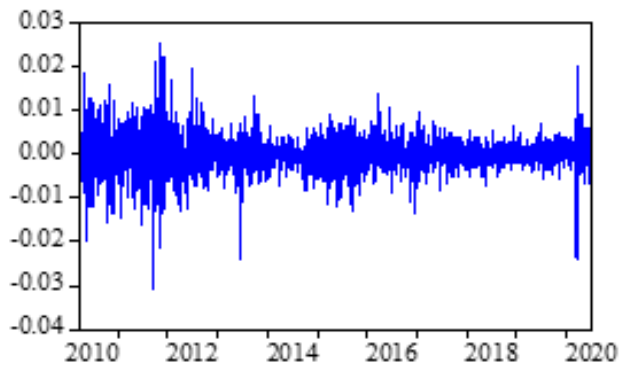

ERIX

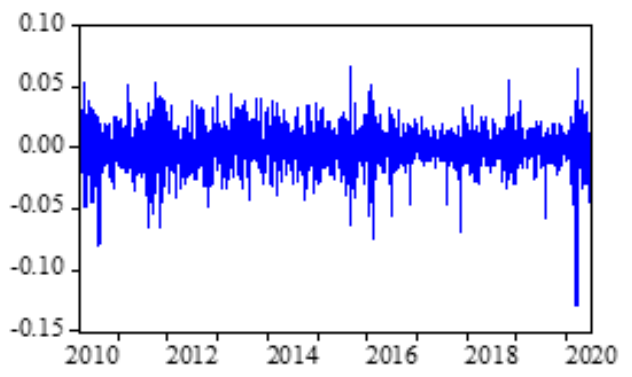

TECH

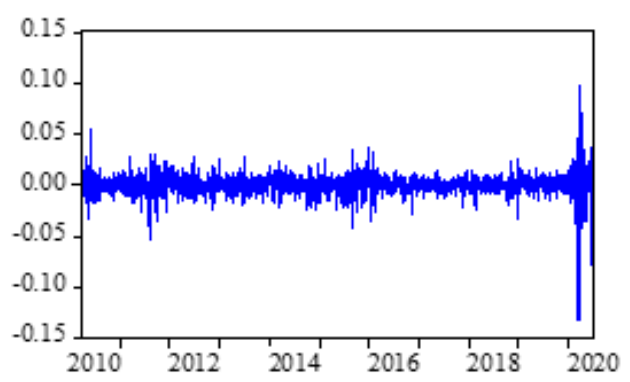

ECO

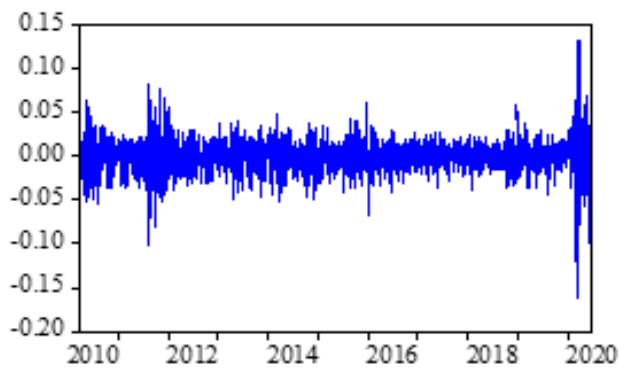

WIND

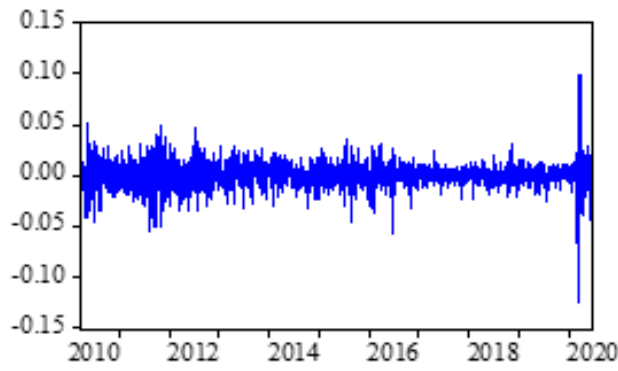

GCE

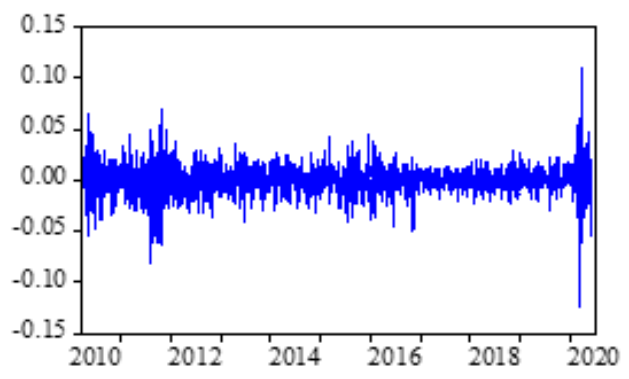

SOLAR

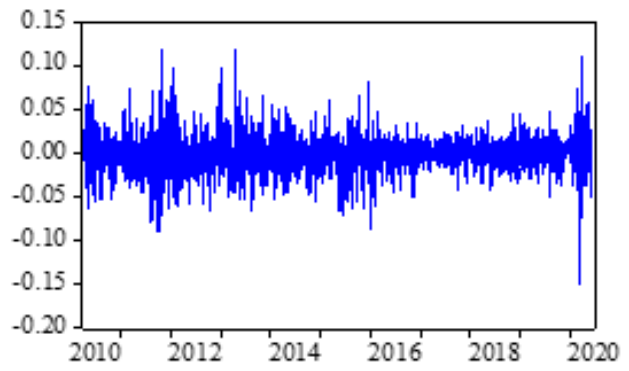

Figure 2

Time-series plot of green bonds and renewable energy returns. 


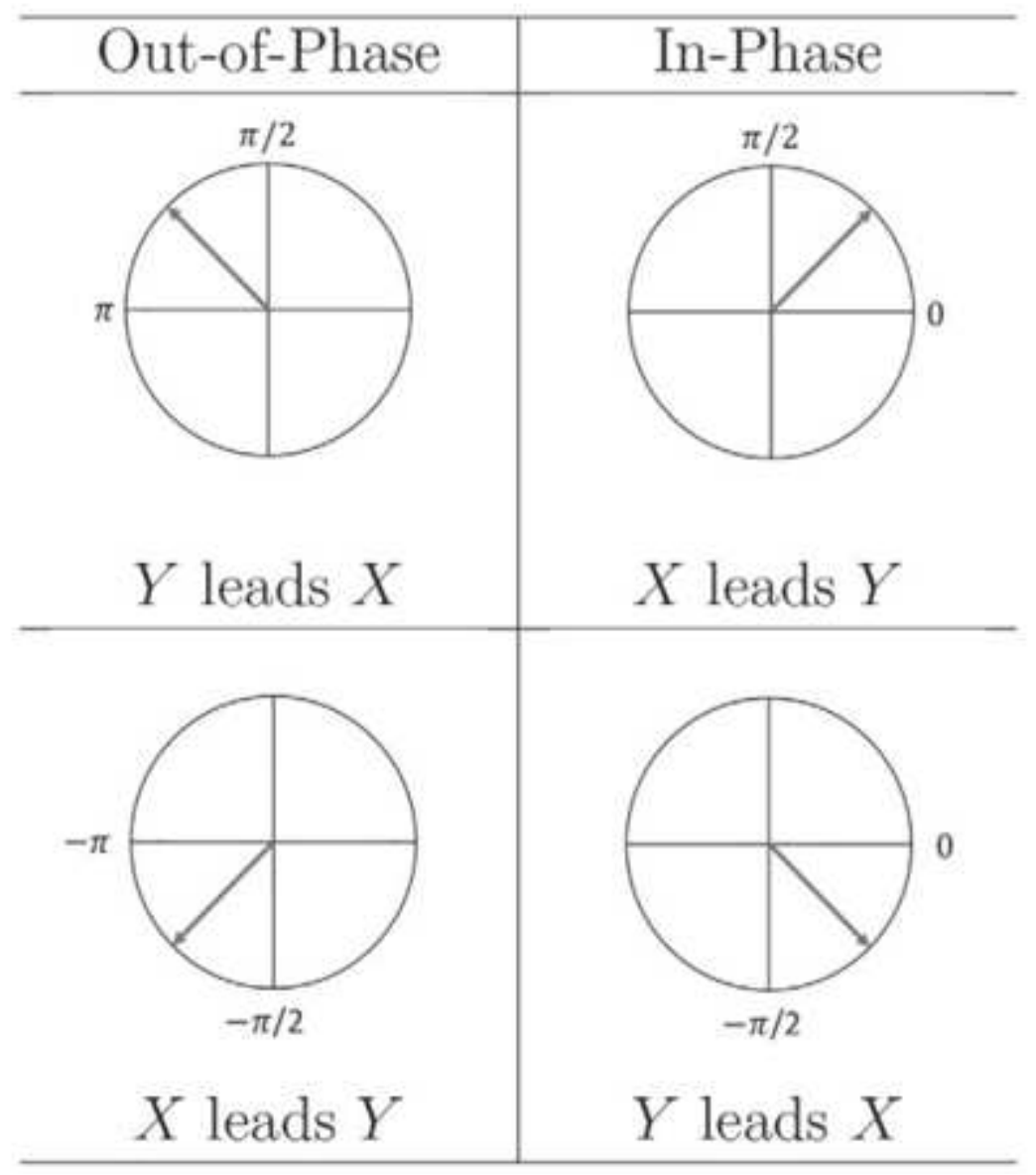

Figure 3

Phase interpretation 


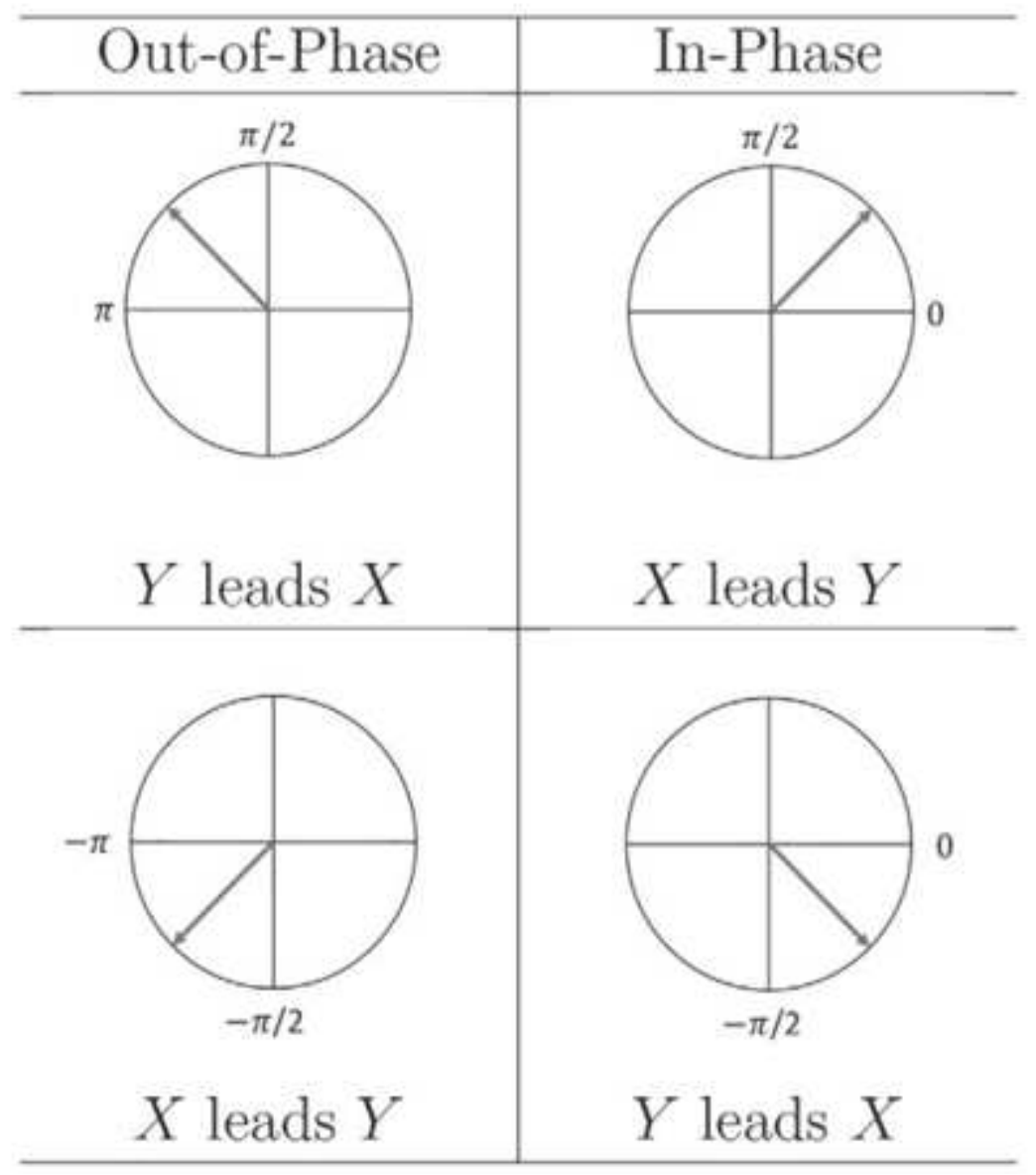

Figure 3

Phase interpretation 

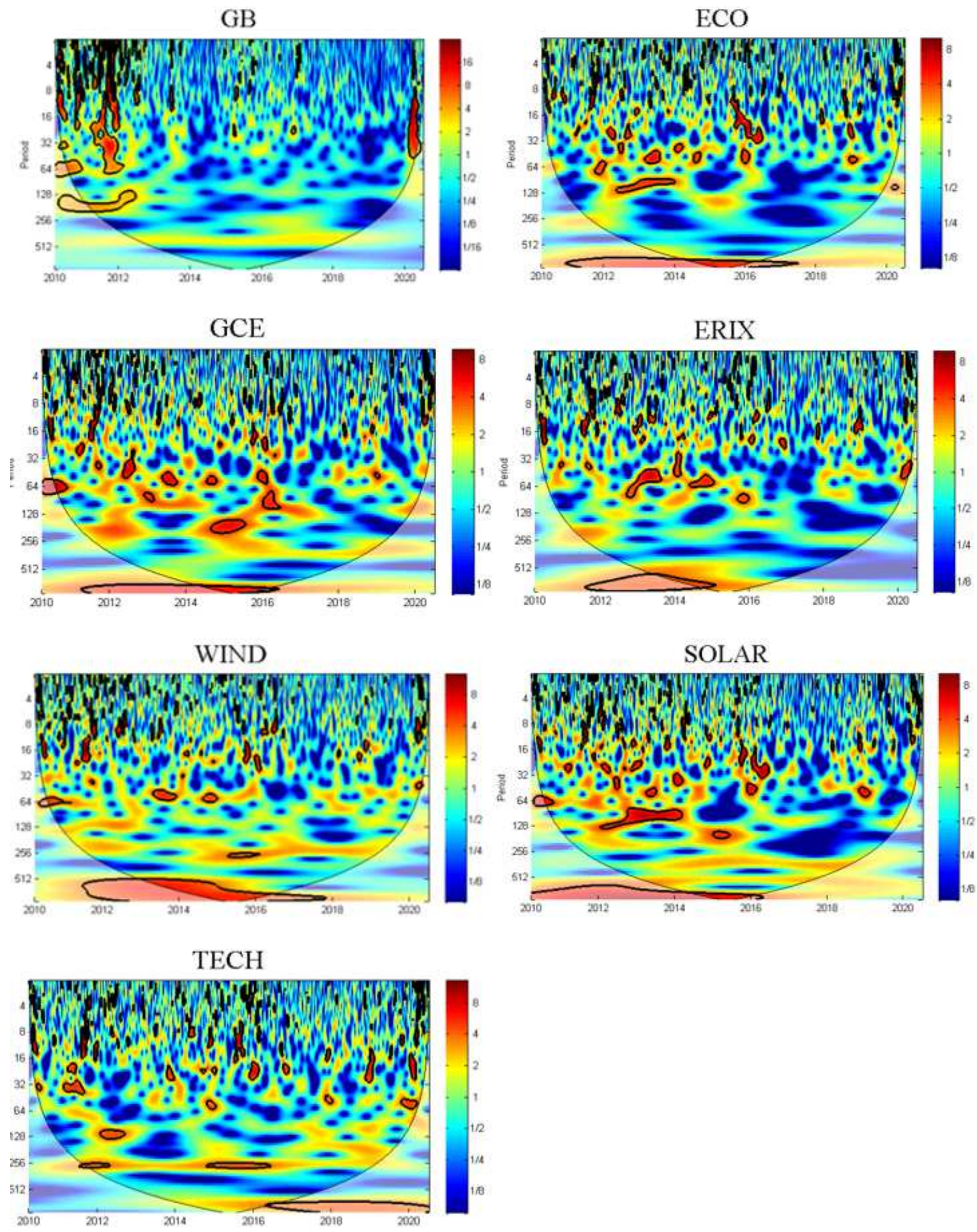

Figure 4

Continuous wavelet transforms for the green bonds and six renewable energy markets. Note: The dark red (blue) indicates strong (smooth) fluctuations and the bold black outline indicates the wavelet power spectrum generated from the Monte Carlo simulation of the $5 \%$ significance level. The region affected by the edge effect is represented by the black curve and defines the cone of influence. The horizontal axis indicates time (year) and the vertical axis indicates period (day). 

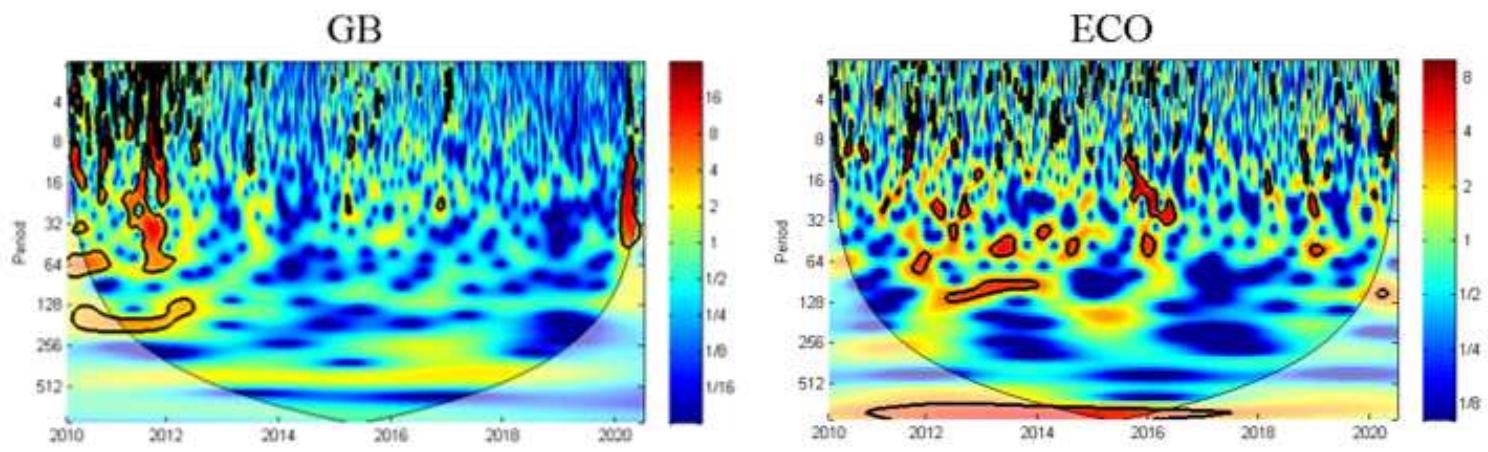

GCE
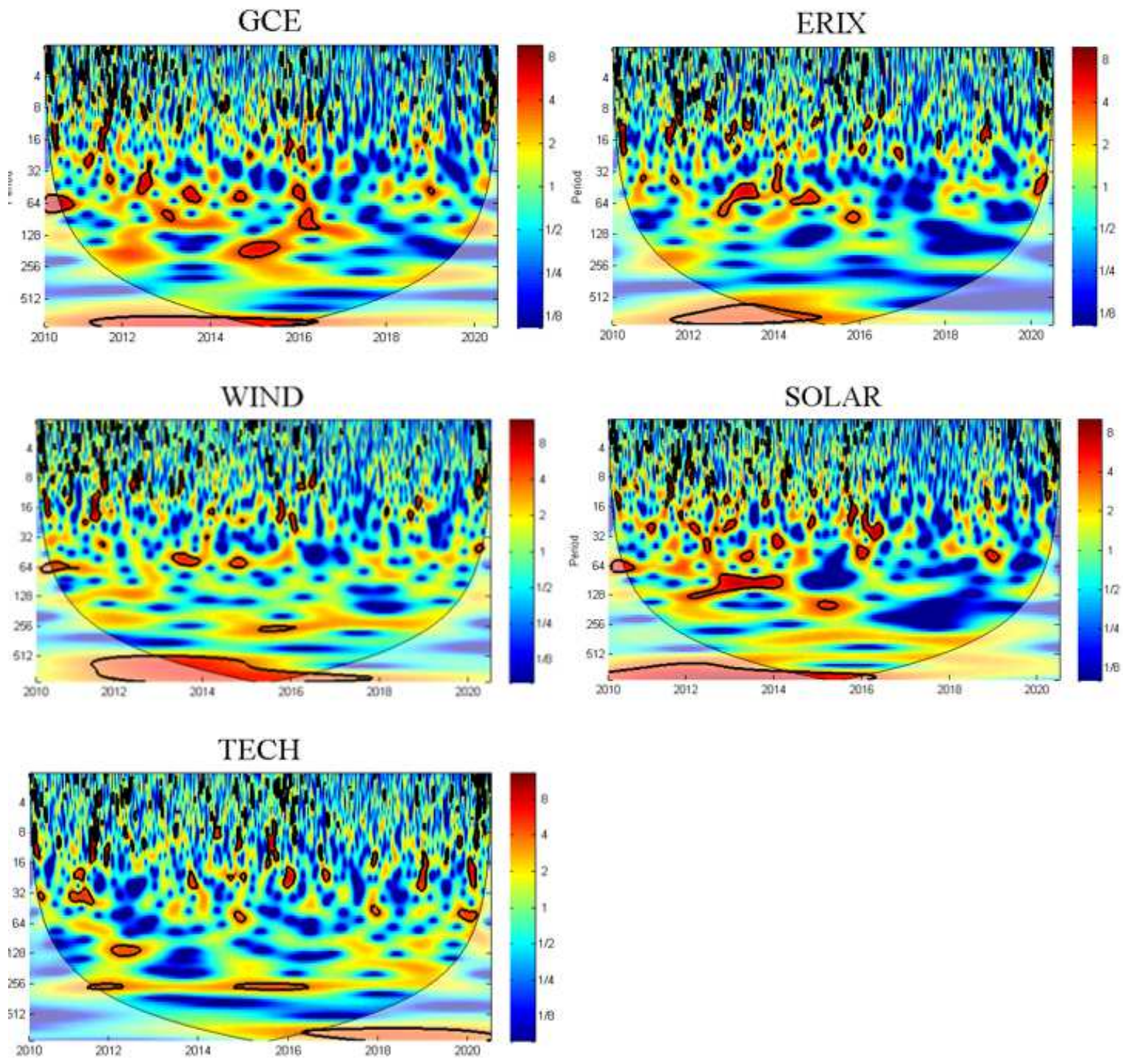

Figure 4

Continuous wavelet transforms for the green bonds and six renewable energy markets. Note: The dark red (blue) indicates strong (smooth) fluctuations and the bold black outline indicates the wavelet power spectrum generated from the Monte Carlo simulation of the $5 \%$ significance level. The region affected by the edge effect is represented by the black curve and defines the cone of influence. The horizontal axis indicates time (year) and the vertical axis indicates period (day). 

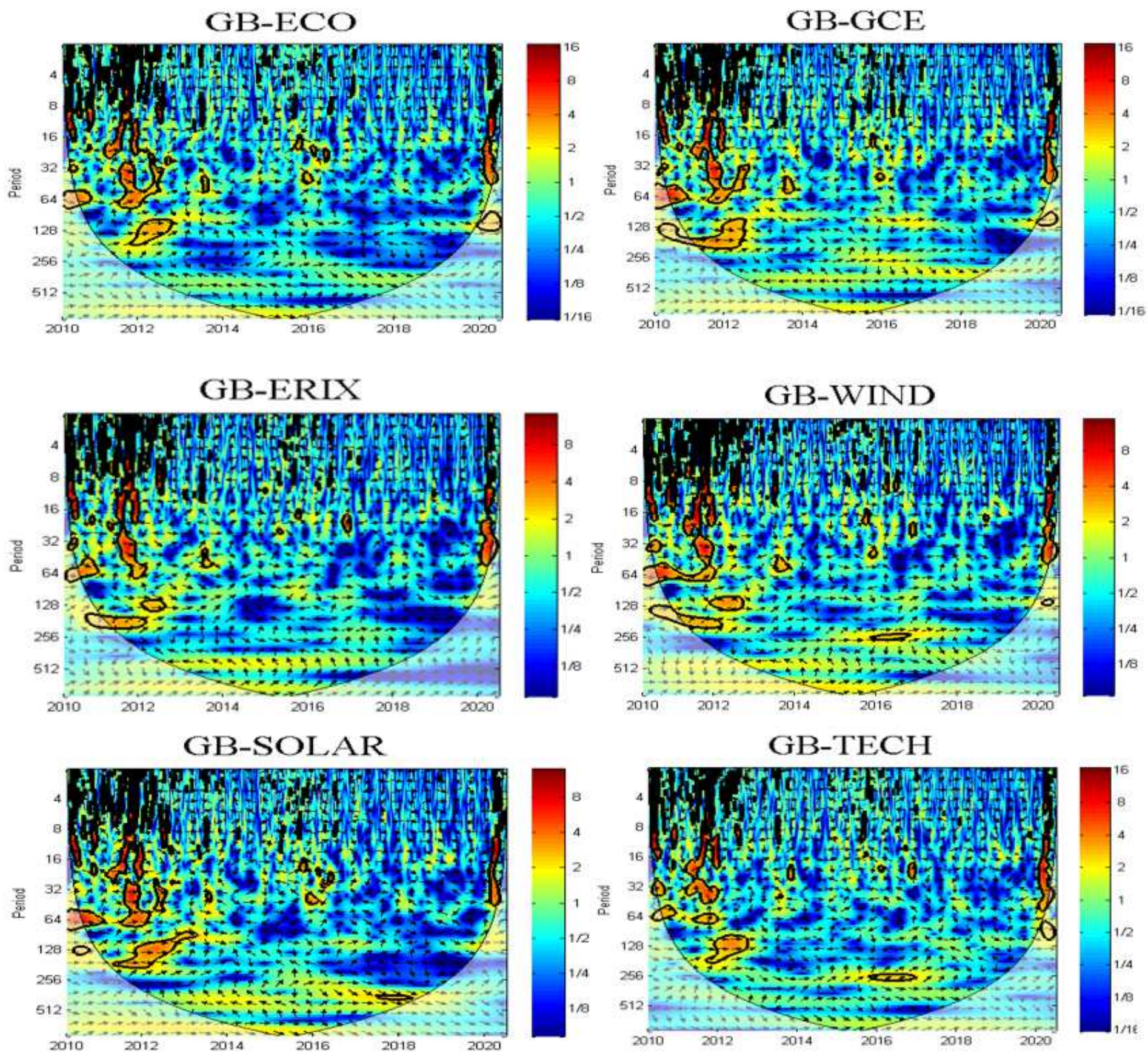

\section{Figure 5}

Cross-wavelet transforms for green bonds and renewable energy indices. Note: the horizontal axis presents time and the vertical axis shows frequency (days). The warmer color of the region, the higher the dependence between the pairs. 

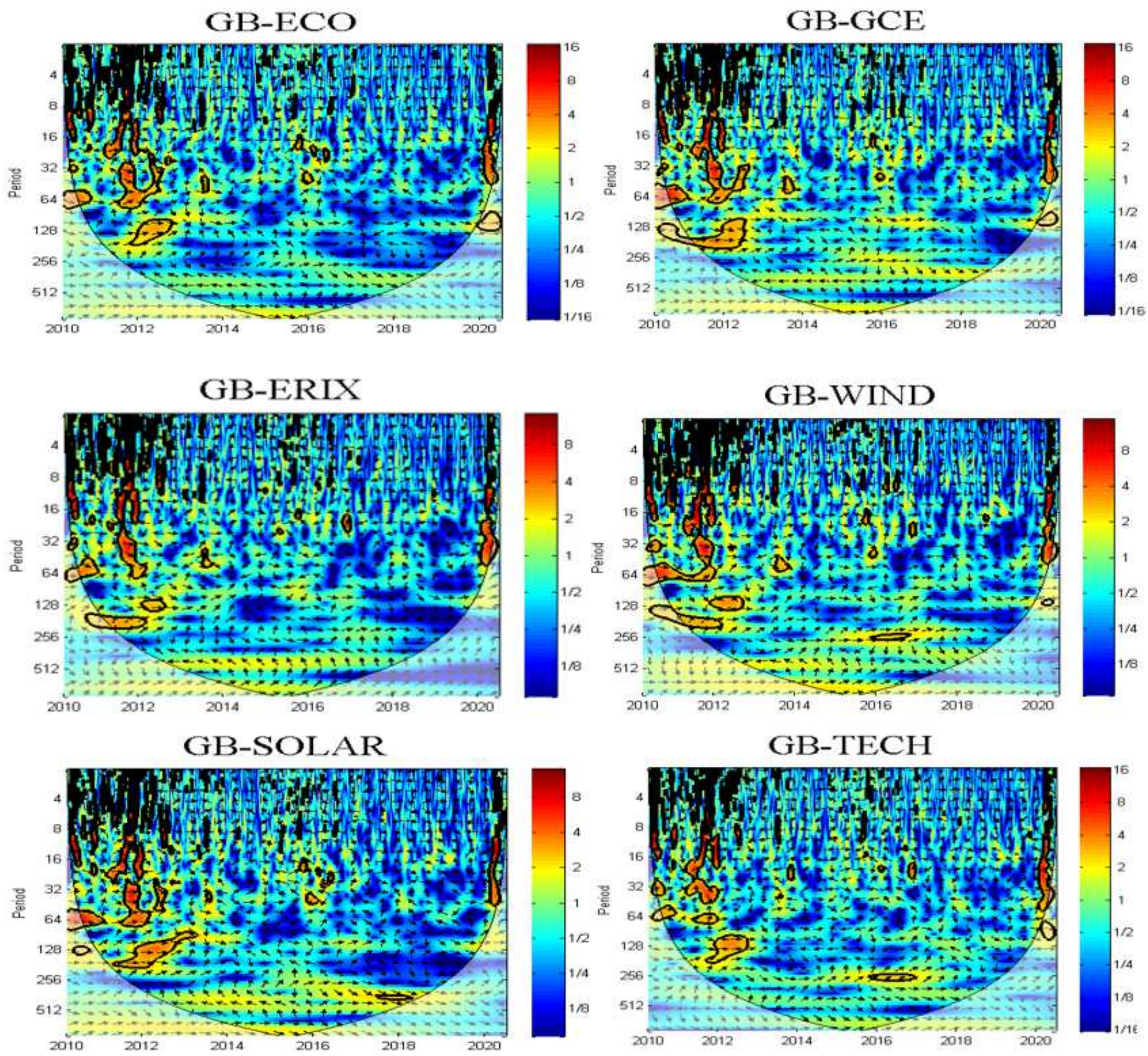

\section{Figure 5}

Cross-wavelet transforms for green bonds and renewable energy indices. Note: the horizontal axis presents time and the vertical axis shows frequency (days). The warmer color of the region, the higher the dependence between the pairs. 

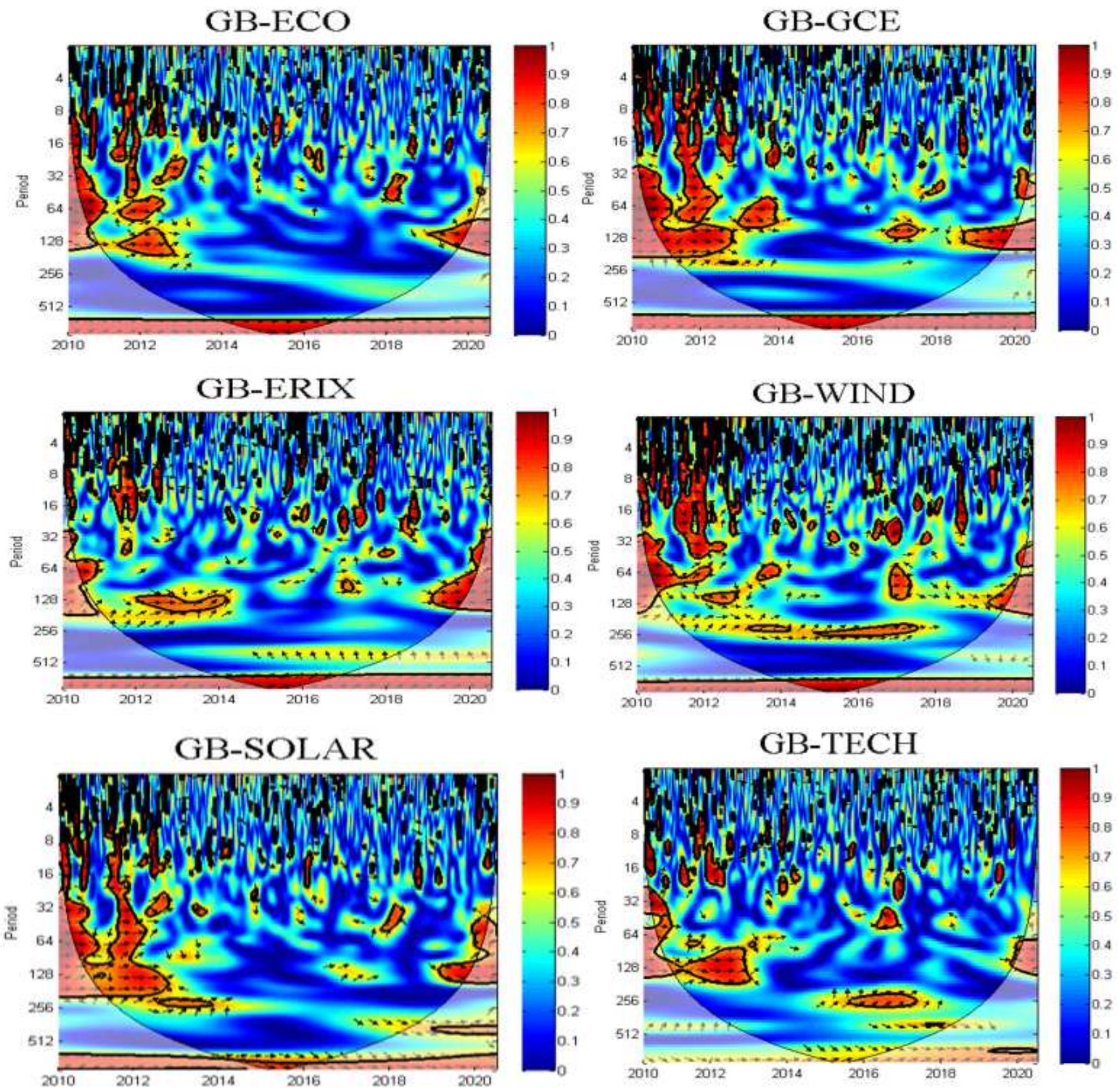

Figure 6

Wavelet coherence of green bonds and renewable energy pairs. Note: refer to Fig.4. 

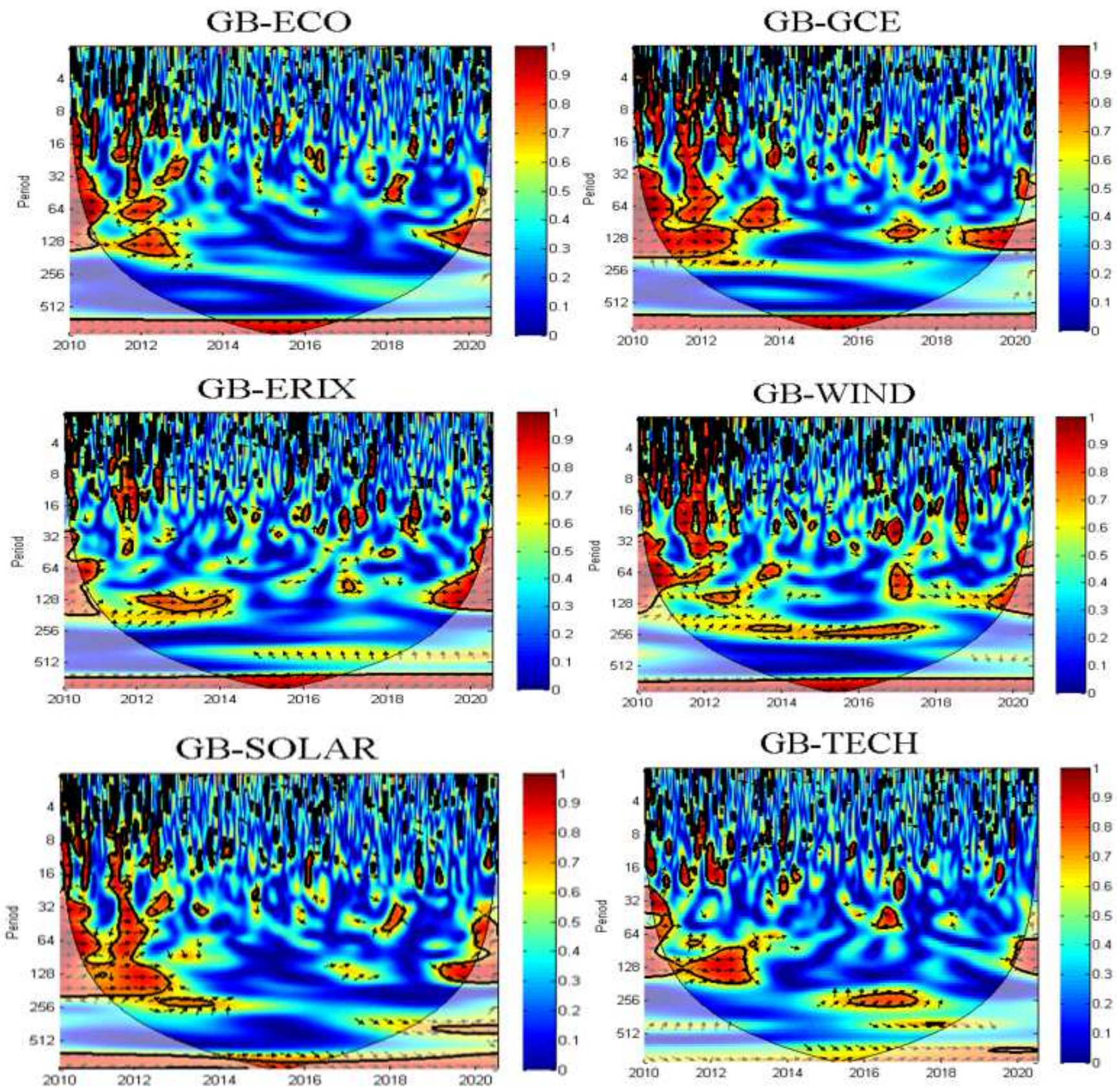

Figure 6

Wavelet coherence of green bonds and renewable energy pairs. Note: refer to Fig.4.

\section{Supplementary Files}

This is a list of supplementary files associated with this preprint. Click to download.

- data.xlsx

- data.xlsx 